\title{
The chemical structure of the very young starless core L1521E $\mathrm{E}^{\star \star \star}$
}

\author{
Z. Nagy ${ }^{1}$, S. Spezzano ${ }^{1}$, P. Caselli ${ }^{1}$, A. Vasyunin ${ }^{2,3}$, M. Tafalla ${ }^{4}$, L. Bizzocchi ${ }^{1}$, D. Prudenzano ${ }^{1}$, and E. Redaelli ${ }^{1}$ \\ ${ }^{1}$ Centre for Astrochemical Studies, Max-Planck-Institute for Extraterrestrial Physics, Giessenbachstrasse 1, 85748 Garching, Germany \\ e-mail: znagy@mpe.mpg.de \\ ${ }^{2}$ Ural Federal University, Ekaterinburg 620002, Russia \\ ${ }^{3}$ Visiting Leading Researcher, Engineering Research Institute, Ventspils International Radio Astronomy Centre of Ventspils \\ University of Applied Sciences, Inženieru 101, Ventspils 3601, Latvia \\ ${ }^{4}$ Observatorio Astronómico Nacional (IGN), Calle Alfonso XII, 3 Madrid, Spain
}

Received 28 March 2019 / Accepted 30 July 2019

\begin{abstract}
Context. L1521E is a dense starless core in Taurus that was found to have relatively low molecular depletion by earlier studies, thus suggesting a recent formation.

Aims. We aim to characterize the chemical structure of L1521E and compare it to the more evolved L1544 pre-stellar core. Methods. We have obtained $\sim 2.5 \times 2.5$ arcminute maps toward L1521E using the IRAM-30 $\mathrm{m}$ telescope in transitions of various species, including $\mathrm{C}^{17} \mathrm{O}, \mathrm{CH}_{3} \mathrm{OH}, c-\mathrm{C}_{3} \mathrm{H}_{2}, \mathrm{CN}, \mathrm{SO}, \mathrm{H}_{2} \mathrm{CS}$, and $\mathrm{CH}_{3} \mathrm{CCH}$. We derived abundances for the observed species and compared them to those obtained toward $\mathrm{L} 1544$. We estimated $\mathrm{CO}$ depletion factors using the $\mathrm{C}^{17} \mathrm{O}$ IRAM-30 m map, an $N\left(\mathrm{H}_{2}\right)$ map derived from Herschel/SPIRE data and a $1.2 \mathrm{~mm}$ dust continuum emission map obtained with the IRAM-30 m telescope.

Results. Similarly to $\mathrm{L} 1544, c-\mathrm{C}_{3} \mathrm{H}_{2}$ and $\mathrm{CH}_{3} \mathrm{OH}$ peak at different positions. Most species peak toward the $c$ - $\mathrm{C}_{3} \mathrm{H}_{2}$ peak including $\mathrm{C}_{2} \mathrm{~S}, \mathrm{C}_{3} \mathrm{~S}, \mathrm{HCS}^{+}, \mathrm{HC}_{3} \mathrm{~N}, \mathrm{H}_{2} \mathrm{CS}, \mathrm{CH}_{3} \mathrm{CCH}$, and $\mathrm{C}^{34} \mathrm{~S}$. $\mathrm{C}^{17} \mathrm{O}$ and $\mathrm{SO}$ peak close to both the $c-\mathrm{C}_{3} \mathrm{H}_{2}$ and the $\mathrm{CH}_{3} \mathrm{OH}$ peaks. $\mathrm{CN}^{-}$and $\mathrm{N}_{2} \mathrm{H}^{+}$ peak close to the Herschel dust peak. We found evidence of CO depletion toward L1521E. The lower limit of the CO depletion factor derived toward the Herschel dust peak is $4.3 \pm 1.6$, which is about a factor of three lower than toward L1544. We derived abundances for several species toward the dust peaks of L1521E and L1544. The abundances of most sulfur-bearing molecules such as $\mathrm{C}_{2} \mathrm{~S}, \mathrm{HCS}^{+}$, $\mathrm{C}^{34} \mathrm{~S}, \mathrm{C}^{33} \mathrm{~S}$, and $\mathrm{HCS}^{+}$are higher toward L1521E than toward L1544 by factors of $\sim 2-20$, compared to the abundance of $\mathrm{A}-\mathrm{CH}_{3} \mathrm{OH}$. The abundance of methanol is very similar toward the two cores.

Conclusions. The fact that the abundances of sulfur-bearing species toward L1521E are higher than toward L1544 suggests that significant sulfur depletion takes place during the dynamical evolution of dense cores, from the starless to pre-stellar stage. The CO depletion factor measured toward L1521E suggests that $\mathrm{CO}$ is more depleted than previously found. Similar $\mathrm{CH}_{3} \mathrm{OH}$ abundances between $\mathrm{L} 1521 \mathrm{E}$ and L1544 hint that methanol is forming at specific physical conditions in the Taurus Molecular Cloud Complex, characterized by densities of a few $\times 10^{4} \mathrm{~cm}^{-3}$ and $N\left(\mathrm{H}_{2}\right) \gtrsim 10^{22} \mathrm{~cm}^{-2}$, when $\mathrm{CO}$ starts to catastrophically freeze-out, while water can still be significantly photodissociated, so that the surfaces of dust grains become rich in solid $\mathrm{CO}$ and $\mathrm{CH}_{3} \mathrm{OH}$, as already found toward $\mathrm{L} 1544$. Methanol can thus provide selective crucial information about the transition region between dense cores and the surrounding parent cloud.
\end{abstract}

Key words. ISM: molecules - radio lines: ISM - ISM: clouds

\section{Introduction}

Starless cores with temperatures of about $10 \mathrm{~K}$ and densities above $10^{4} \mathrm{~cm}^{-3}$ define the stage just before the onset of low-mass star formation in molecular clouds. When the central $\mathrm{H}_{2}$ density of the cores is above $10^{5} \mathrm{~cm}^{-3}$, starless cores become thermally supercritical and start to collapse. These are the so-called prestellar cores. Probing the physics and chemistry of such regions is essential for understanding the process of star formation. The chemistry of starless and pre-stellar cores can be traced by different molecular lines and the dust continuum at millimetre and sub-millimetre wavelengths. In this paper we aim to characterize the chemical structure of a starless core in an early evolutionary phase.

The L1521E starless core is located in the Taurus region, at a distance of $145_{-16}^{+12}$ pc (Yan et al. 2019). It was classified as

\footnotetext{
* The reduced datacubes are also available at the CDS via anonymous ftp to cdsarc.u-strasbg. fr (130.79.128.5) or via http:// cdsarc.u-strasbg.fr/viz-bin/cat/J/A+A/630/A136

$\star \star$ Based on observations carried out with the IRAM $30 \mathrm{~m}$ telescope IRAM is supported by INSU/CNRS (France), MPG (Germany) and IGN (Spain).
}

a very young core by Hirota et al. (2002) because of the high abundances of carbon-chain molecules, comparable to those in TMC-1 (Aikawa et al. 2003). Tafalla \& Santiago (2004) further confirmed that L1521E was a very young core as they found no evidence of $\mathrm{C}^{18} \mathrm{O}$ depletion. They estimate its age to be $\leq 1.5 \times$ $10^{5}$ yr. Ford \& Shirley (2011) confirmed the low level of CO depletion by fitting $\mathrm{C}^{18} \mathrm{O}$ line profiles using a non-LTE radiative transfer code. Hirota et al. (2002) derived the peak $\mathrm{H}_{2}$ volume density to be $(1.3-5.6) \times 10^{5} \mathrm{~cm}^{-3}$, which is consistent with the value of $2.7 \times 10^{5} \mathrm{~cm}^{-3}$ found by Tafalla \& Santiago (2004). Based on Herschel/SPIRE observations, Makiwa et al. (2016) measured the dust temperature to be $9.8 \pm 0.2 \mathrm{~K}$ and the core mass to be $1.0 \pm 0.1 M_{\odot}$.

In this paper we aim to study the chemical structure and $\mathrm{CO}$ depletion of L1521E and compare it to the more evolved and more massive pre-stellar core L1544. L1544 is a well-studied pre-stellar core also located in Taurus (Spezzano et al. 2017 and references therein). Based on a comparison of observations and chemical models of simple deuterated species toward L1544, Kong et al. (2015) derived an age of a few $10^{5}$ yr. Based on $1.2 \mathrm{~mm}$ contimuum data, the $N\left(\mathrm{H}_{2}\right)$ toward the dust peak is $(9.4 \pm 1.6) \times 10^{22} \mathrm{~cm}^{-2}$ and the $\mathrm{H}_{2}$ volume density within a 

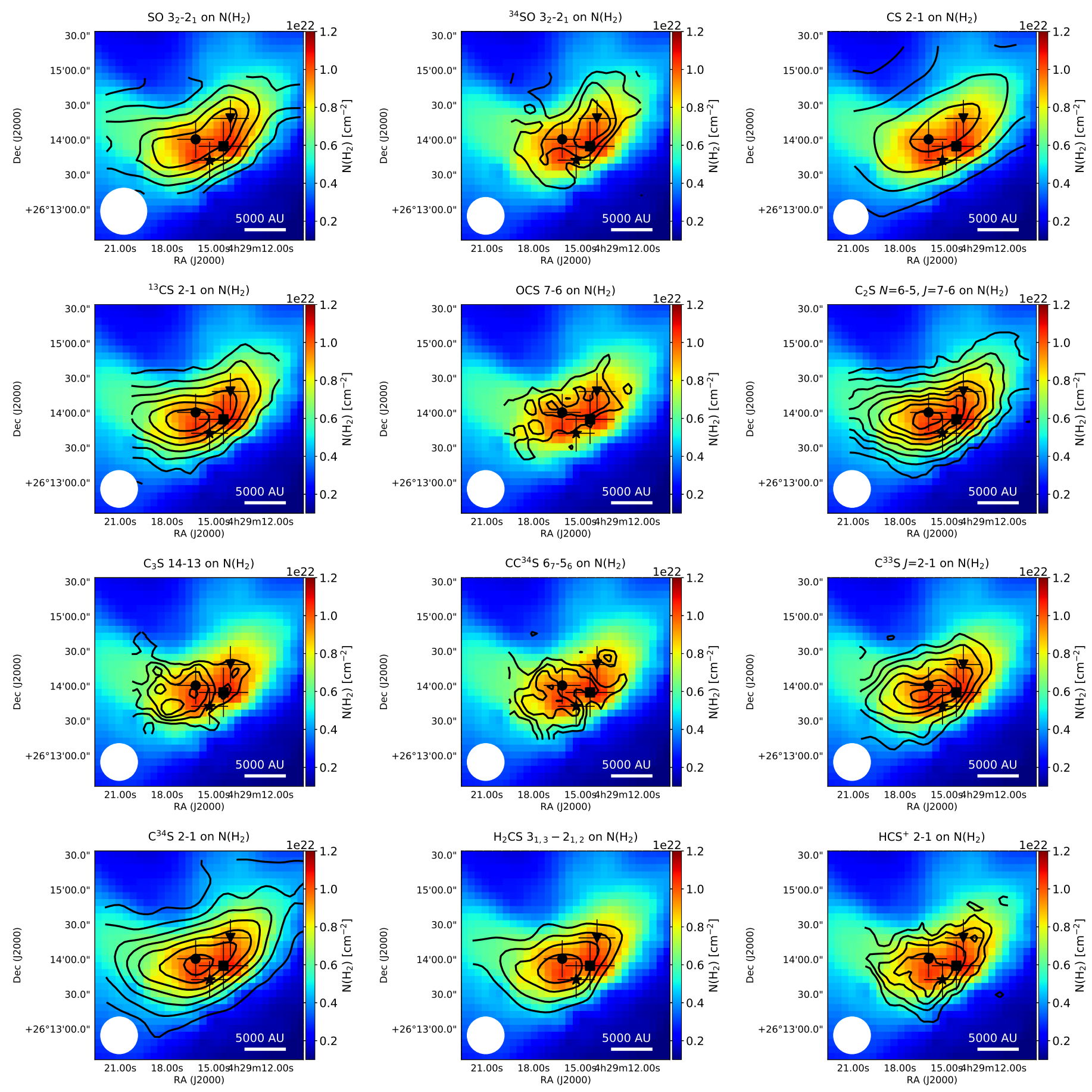

Fig. 1. Spatial distributions of molecules detected toward L1521E (black contours) overplotted on $N\left(\mathrm{H}_{2}\right)$ map (colors) derived from Herschel/SPIRE. The black dot, triangle, and asterisk show the $c-\mathrm{C}_{3} \mathrm{H}_{2}, \mathrm{CH}_{3} \mathrm{OH}$, and $\mathrm{HNCO}$ peaks, respectively. The black square shows the Herschel dust peak. The beam size overplotted on the SO map shows the beam size of the SPIRE data, for the other maps it is the beam size of the IRAM-30 m data. For SO, CS, ${ }^{13} \mathrm{CS}, \mathrm{C}_{2} \mathrm{~S}$, and $\mathrm{C}^{34} \mathrm{~S}$ the contour levels start from $3 \sigma \mathrm{rms}$ in steps of $3 \sigma$ rms with $3 \sigma$ rms values of $0.13,0.38$, $0.06,0.12$, and $0.11 \mathrm{~K} \mathrm{~km} \mathrm{~s}^{-1}$, respectively. For OCS and $\mathrm{C}^{33} \mathrm{~S}$ the contour levels start from $6 \sigma \mathrm{rms}$ in steps of $4 \sigma$ rms with $3 \sigma$ rms levels of $0.02 \mathrm{~K} \mathrm{~km} \mathrm{~s}^{-1}$ for both species. For ${ }^{34} \mathrm{SO}, \mathrm{C}^{3} \mathrm{~S}, \mathrm{CC}^{34} \mathrm{~S}, \mathrm{H}_{2} \mathrm{CS}$, and $\mathrm{HCS}^{+}$the contour levels start from $9 \sigma \mathrm{rms}$ in steps of $6 \sigma \mathrm{rms}$ with $3 \sigma$ rms values of $0.01,0.02,0.01,0.07$, and $0.03 \mathrm{~K} \mathrm{~km} \mathrm{~s}^{-1}$, respectively.

radius of $(3.2 \pm 0.4) \times 10^{3} \mathrm{AU}$ is $(1.4 \pm 0.2) \times 10^{6} \mathrm{~cm}^{-3}$ (Crapsi et al. 2005; Chacon-Tanarro et al. 2019). Comparing the less evolved L1521E to the more evolved and better characterized L1544 will help us to gain understanding of the evolution of dense cores, and hence the process of star formation. The paper is organized as follows: we describe the observations in Sect. 2 and in Sect. 3. Results on the spatial distribution and column densities of the molecules, and on CO depletion are presented in
Sect. 4. We discuss the results and compare them to those toward L1544 in Sect. 5 and summarize them in Sect. 6.

\section{Observations and data reduction}

Maps of various molecules were observed using the Eight MIxer Receiver (EMIR) instrument of the IRAM-30 m telescope in on-the-fly mode with position switching. The Fourier transform 

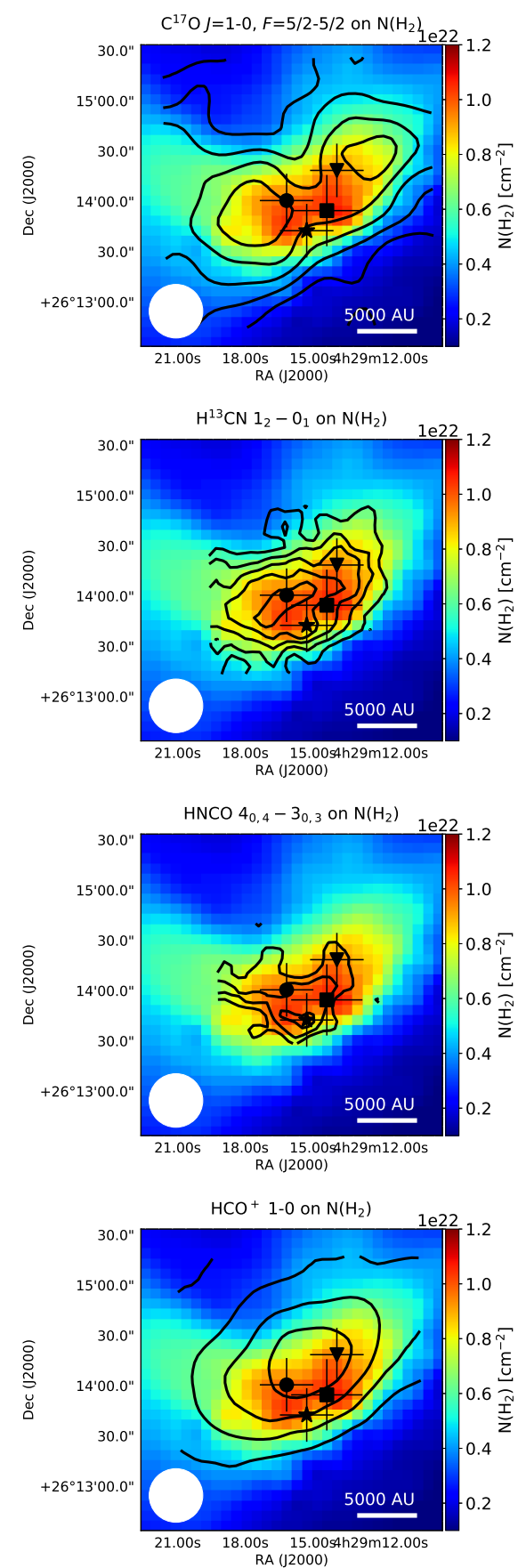
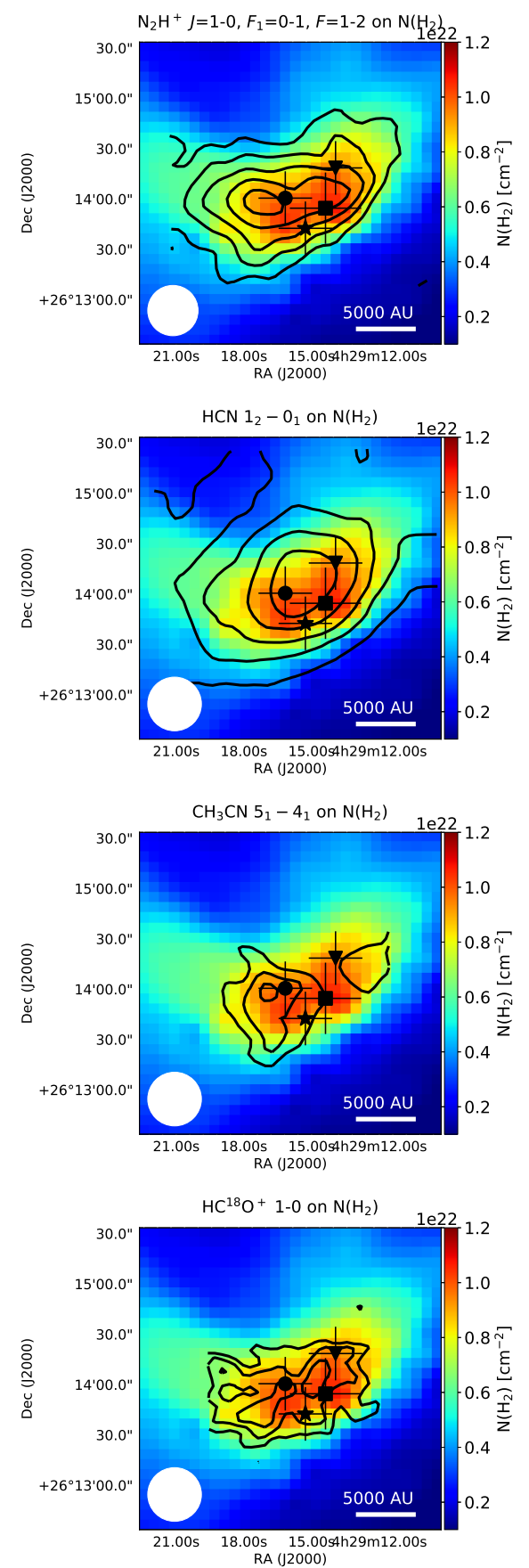
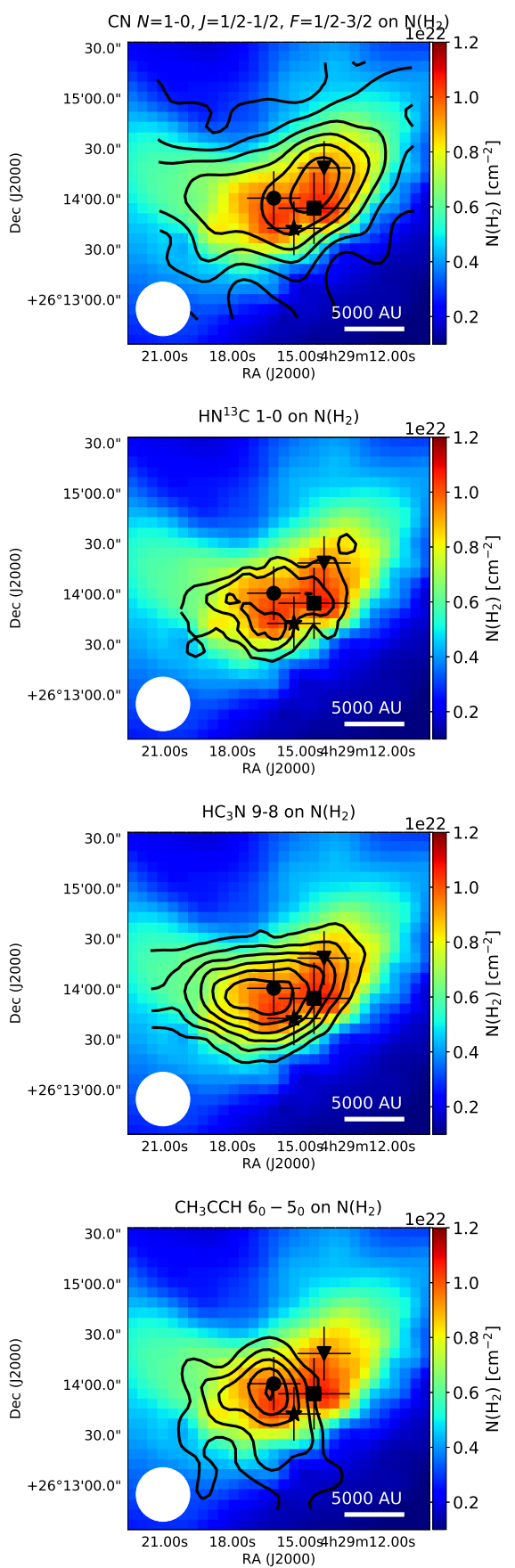

Fig. 1. continued. For $\mathrm{C}^{17} \mathrm{O}, \mathrm{CN}, \mathrm{H}^{13} \mathrm{CN}, \mathrm{HCN}$, and $\mathrm{HCO}^{+}$the contour levels start from 3- $\sigma$ rms in steps of 3- $\sigma$ rms with 3- $\sigma$ rms values of $0.05,0.05,0.04,0.17$, and $0.34 \mathrm{~K} \mathrm{~km} \mathrm{~s}^{-1}$, respectively. For $\mathrm{N}_{2} \mathrm{H}^{+}, \mathrm{HN}^{13} \mathrm{C}, \mathrm{HNCO}, \mathrm{HC}_{3} \mathrm{~N}$, and $\mathrm{HC}^{18} \mathrm{O}^{+}$the contour levels start from 6- $\sigma$ rms in steps of 4- $\sigma$ rms with 3- $\sigma$ rms levels of $0.08,0.08,0.05,0.12$, and $0.02 \mathrm{~K} \mathrm{~km} \mathrm{~s}^{-1}$, respectively. For $\mathrm{CH}_{3} \mathrm{CN}$ and $\mathrm{CH}_{3} \mathrm{CCH}$ the contour levels start from $9-\sigma \mathrm{rms}$ in steps of 6- $\sigma \mathrm{rms}$ with $3-\sigma \mathrm{rms}$ values of 0.01 and $0.03 \mathrm{~K} \mathrm{~km} \mathrm{~s}^{-1}$, respectively.

spectrometer (FTS) was used as the backend with a spectral resolution of $50 \mathrm{kHz}$. The $4-6.25$ square arcminute maps were centered on the $\mathrm{RA}(\mathrm{J} 2000)=04^{\mathrm{h}} 29^{\mathrm{m}} 15.7^{\mathrm{s}} \operatorname{Dec}(\mathrm{J} 2000)=+26^{\circ} 14^{\prime} 5^{\prime \prime}$ position, based on Tafalla \& Santiago (2004). Part of the observations were carried out in June and August 2016 (PI: S. Spezzano), and additional observations were done in 2018 January and April (PI: Z. Nagy). The data were reduced using the GILDAS software (Pety 2005). The measured intensities were converted to main beam temperature units using a forward efficiency of 0.94 and a main beam efficiency of 0.78 around the frequency of $115 \mathrm{GHz}$, and a forward efficiency of 0.95 and a beam efficiency of 0.81 around the frequency of $86 \mathrm{GHz}$. At these frequencies, the angular resolution of the IRAM- $30 \mathrm{~m}$ observations is around 30". When creating the data cubes analyzed in this paper we re-gridded the maps to a pixel size of $4^{\prime \prime}$, as done by Spezzano et al. (2017).

We use Herschel/SPIRE (Griffin et al. 2010) data from bands at 250,350 , and $500 \mu \mathrm{m}$ to derive the $\mathrm{H}_{2}$ column densities. These data were downloaded from the Herschel Science Archive and are part of the Herschel Gould Belt Survey (André et al. 2010). We fit a modified blackbody function with a dust emissivity index of $\beta=1.5$ to each pixel. The 250 and $350 \mu \mathrm{m}$ images were smoothed to the resolution of the $500 \mu \mathrm{m}$ image $\left(\sim 40^{\prime \prime}\right)$. The images were resampled to the same grid.

In addition to the IRAM-30 $\mathrm{m}$ and Herschel/SPIRE data we also used the $1.2 \mathrm{~mm}$ continuum data from the work of 

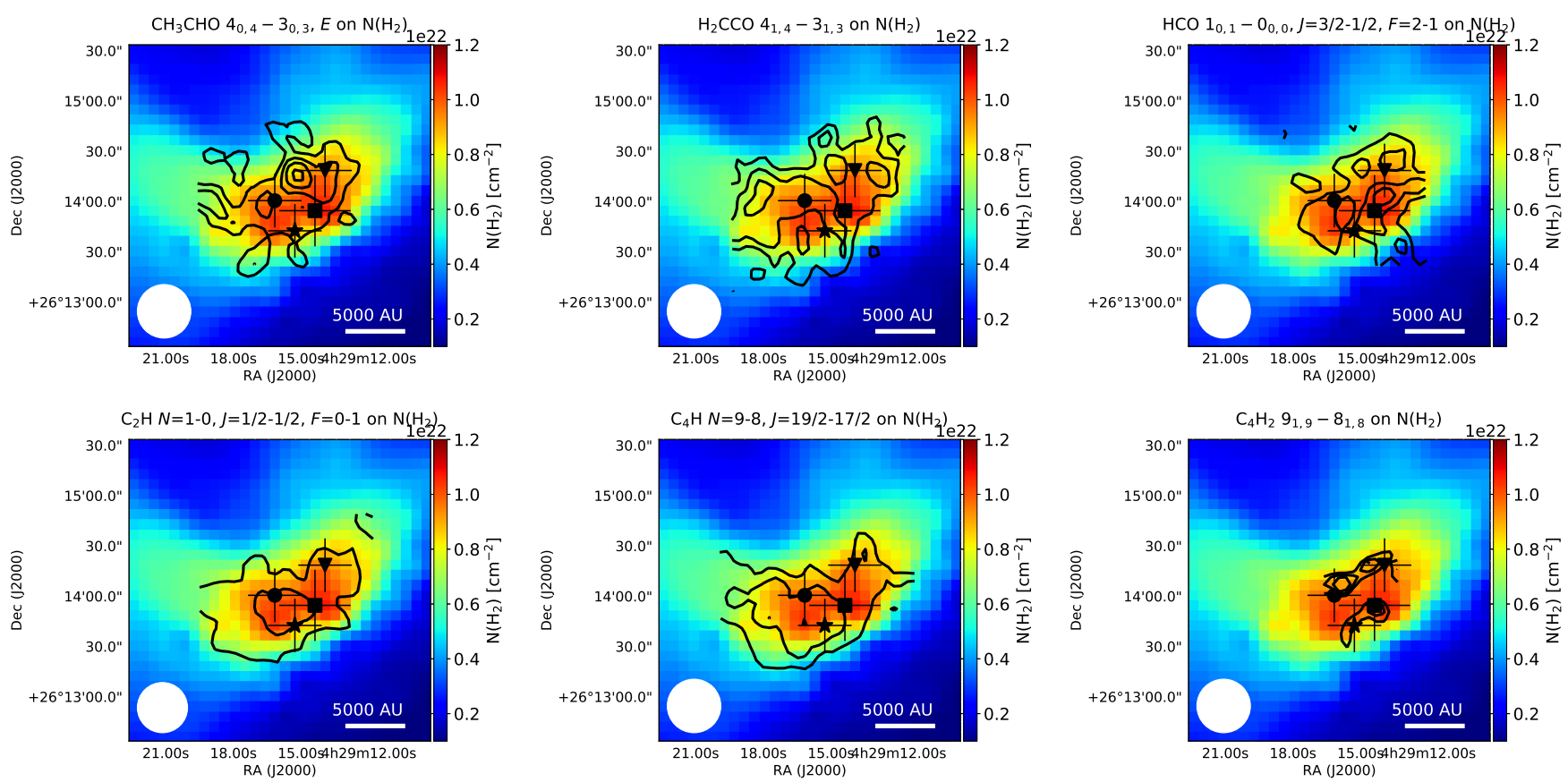

Fig. 1. continued. For $\mathrm{H}_{2} \mathrm{CCO}$ and $\mathrm{HCO}$ the contour levels start from $6-\sigma \mathrm{rms}$ in steps of 4- $\sigma$ rms with 3- $\sigma$ rms levels of $0.02 \mathrm{~K} \mathrm{~km} \mathrm{~s}^{-1}$ for both species. For $\mathrm{CH}_{3} \mathrm{CHO}, \mathrm{C}_{2} \mathrm{H}, \mathrm{C}_{4} \mathrm{H}$, and $\mathrm{C}_{4} \mathrm{H}_{2}$ the contour levels start from 9- $\sigma$ rms in steps of $6-\sigma \mathrm{rms}$ with 3- $\sigma$ rms values of $0.01,0.07,0.03$, and $0.004 \mathrm{~K} \mathrm{~km} \mathrm{~s}^{-1}$, respectively.

Tafalla \& Santiago (2004). These data were measured with the MAMBO-2 instrument of the IRAM- $30 \mathrm{~m}$ telescope in on-thefly mode. The spatial resolution of the $1.2 \mathrm{~mm}$ continuum data is $11^{\prime \prime}$.

\section{Dataset}

The dataset analyzed in this paper includes maps of molecular emission from 30 species (including isotopologs) detected toward L1521E as shown in Table A.1. Some species, such as isotopologs containing ${ }^{15} \mathrm{~N}$ and deuterated species will be analyzed in a forthcoming paper. The quantum numbers, frequencies, Einstein $A$ coefficients, and upper level energies are from the Cologne Database for Molecular Spectroscopy (CDMS, Müller et al. 2005) ${ }^{1}$ and the Jet Propulsion Laboratory (JPL, Pickett et al. 1998) $)^{2}$ molecular databases. We included the critical densities for the transitions whenever collisional rates are available for the molecules. Table A.1 also includes information on whether the transitions detected toward L1521E are detected toward L1544. This information is based on the public data release of the Astrochemical Surveys At IRAM (ASAI, Lefloch et al. 2018; Vastel et al. 2018). A spectrum in the frequency range between 80 and $106 \mathrm{GHz}$ was observed toward the dust peak of L1544, which allows us to directly compare the two sources in Sect. 5. Figure 1 shows integrated intensity maps of the molecules detected toward L1521E, which will be further discussed in the next sections.

\section{Results}

\subsection{Spatial distribution of the molecules}

Integrated intensity maps of the detected species are shown in Figs. 1 and 2. The spatial distribution of the different species is further shown as line profiles extracted toward the $c-\mathrm{C}_{3} \mathrm{H}_{2}$ and methanol peaks in Fig. 3. Furthermore, shown in Fig. 4

\footnotetext{
1 https://cdms.astro.uni-koeln.de

2 http://spec.jpl.nasa.gov
}

are intensity cuts extracted toward a line that crosses the two molecular peaks, and toward a line that is perpendicular to the former and then crosses the Herschel dust peak. The position of the two cuts is shown in Fig. 2. Similar to what was observed in the more evolved L1544 core (Spezzano et al. 2016), methanol and $c-\mathrm{C}_{3} \mathrm{H}_{2}$ peak at different positions around the center of L1521E (Fig. 2). Most species peak where $c-\mathrm{C}_{3} \mathrm{H}_{2}$ peaks $\left(\mathrm{RA}(\mathrm{J} 2000)=04^{\mathrm{h}} 29^{\mathrm{m}} 16.0^{\mathrm{s}} \operatorname{Dec}(\mathrm{J} 2000)=+26^{\circ} 14^{\prime} 0.9^{\prime \prime}\right)$, such as $\mathrm{C}_{2} \mathrm{~S}, \mathrm{C}_{3} \mathrm{~S}, \mathrm{HCS}^{+}, \mathrm{HC}_{3} \mathrm{~N}, \mathrm{H}_{2} \mathrm{CS}, \mathrm{CH}_{3} \mathrm{CCH}$, and $\mathrm{C}^{34} \mathrm{~S}$. The $\mathrm{CN}$ emission peak is between the Herschel dust peak and the methanol peak $\left(\operatorname{RA}(J 2000)=04^{\mathrm{h}} 29^{\mathrm{m}} 14.4^{\mathrm{s}}\right.$ $\left.\operatorname{Dec}(\mathrm{J} 2000)=+26^{\circ} 14^{\prime} 17.7^{\prime \prime}\right)$, and considering the Herschel and IRAM-30 m beam sizes, it is consistent with both peaks. $\mathrm{N}_{2} \mathrm{H}^{+}$ also peaks near the Herschel dust peak.

Figure 5 shows the spatial distribution of gas traced by the detected species compared to the location of the dust peak traced by the $1.2 \mathrm{~mm}$ and the Herschel continuum data. The dust peak based on the Herschel/SPIRE data $\left(\mathrm{RA}(\mathrm{J} 2000)=04^{\mathrm{h}} 29^{\mathrm{m}} 14.0^{\mathrm{s}}\right.$ $\left.\operatorname{Dec}(\mathrm{J} 2000)=+26^{\circ} 13^{\prime} 56^{\prime \prime}\right)$ is slightly offset from the $1.2 \mathrm{~mm}$ peak $\left(\mathrm{RA}(\mathrm{J} 2000)=04^{\mathrm{h}} 29^{\mathrm{m}} 15.15^{\mathrm{s}} \operatorname{Dec}(\mathrm{J} 2000)=+26^{\circ} 13^{\prime} 50.8^{\prime \prime}\right)$, but considering the large SPIRE beam, it is consistent with both the $1.2 \mathrm{~mm}$ peak and the $\mathrm{CH}_{3} \mathrm{OH}$ peak. Also, SPIRE is probably probing a different dust population compared to the $1.2 \mathrm{~mm}$ data. The distribution of $\mathrm{N}_{2} \mathrm{H}^{+}$is similar to the distribution of dust, as it is also the case for $\mathrm{L} 1544$ (Caselli et al. 1999). $\mathrm{C}^{17} \mathrm{O}$ shows signs of depletion toward the dust peak, which was also observed for $\mathrm{L} 1544$ (Caselli et al. 1999). $\mathrm{CH}_{3} \mathrm{OH}$ peaks in a region where $\mathrm{CO}$ is depleted, but not exactly towards the dust peak or towards the region where the $\mathrm{CO}$ freeze-out is the most prominent. The spatial distribution of species shown in Fig. 5 and the depletion of $\mathrm{CO}$ is further discussed in Sect. 4.4.

\subsection{Principal component analysis}

Another method to study the structure of L1521E and probe correlations between the observed molecular species is the principal component analysis (PCA), which Spezzano et al. (2017) previously performed for L1544. A detailed description of the 

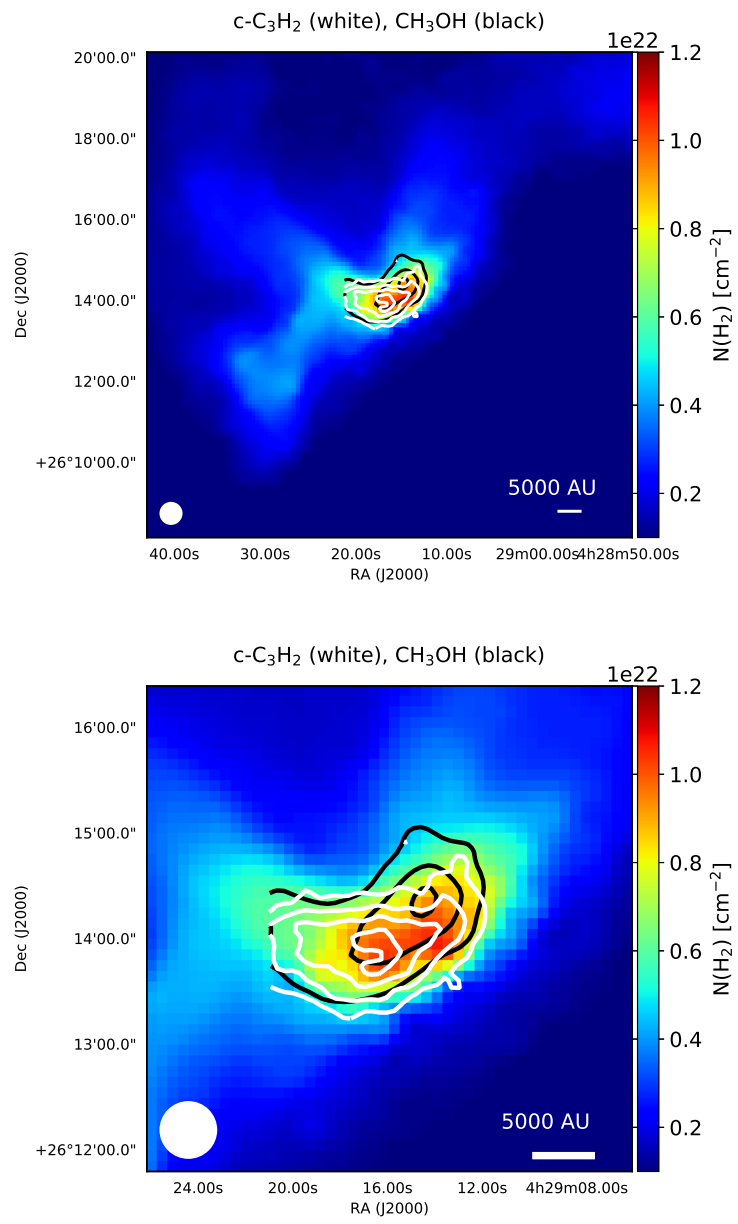

Fig. 2. Spatial distribution of $c-\mathrm{C}_{3} \mathrm{H}_{2} 2_{0,2}-1_{1,1}$ (white contours) and $\mathrm{CH}_{3} \mathrm{OH}\left(E_{2}\right)$ (black contours). These are shown as detected toward L1521E overplotted on the $N\left(\mathrm{H}_{2}\right)$ map which was derived from Herschel/SPIRE (color map), showing the large-scale environment of L1521E (top) and the spatial structure of molecular line emission close to the center of the core (bottom). The two overplotted lines show the location of the cuts along which the line intensities shown in Fig. 4 were extracted. The contour levels start from $6 \sigma \mathrm{rms}$ in steps of $6 \sigma \mathrm{rms}$, with $3 \sigma$ rms levels of 0.07 and $0.06 \mathrm{~K} \mathrm{~km} \mathrm{~s}^{-1}$ for $\mathrm{CH}_{3} \mathrm{OH}$ and $c-\mathrm{C}_{3} \mathrm{H}_{2}$, respectively.

method is shown in Ungerechts et al. (1997). The PCA explains the total variability of correlated variables through the use of the same number of orthogonal principal components. Here the number of correlated variables is the number of maps used for the analysis: 24 . Each map consists of $27 \times 27$ pixels in a space with 24 dimensions (the number of molecular transitions used). We used a transition for most molecular species included in this analysis, except for the species with the lowest signal-to-noise ratio $(\mathrm{S} / \mathrm{N}): \mathrm{C}_{3} \mathrm{~S}, \mathrm{CC}^{34} \mathrm{~S}, \mathrm{CH}_{3} \mathrm{CHO}, \mathrm{HC}^{18} \mathrm{O}^{+}, \mathrm{HCO}, \mathrm{OCS}$, and $\mathrm{C}_{4} \mathrm{H}_{2}$. Similar to Spezzano et al. (2017), we performed the PCA on a standardized (mean-centered and normalized) dataset, with the standardized value defined as $x_{\text {std }}=(x-\mu) / \sigma$, where $\mu$ is the mean value and $\sigma$ is the standard deviation for each map. While it is possible to compute 24 principal components (PCs) from our data, we analyze the first four PCs in this paper, as those account for most of the correlation $(91.3 \%)$ in the data. Figure 6 shows maps of the first four PCs. The first PC correlates with the continuum emission, as shown in Fig. 1. Similar to the case of L1544 (Spezzano et al. 2017), the second PC shows the two most prominent molecular peaks, the $c-\mathrm{C}_{3} \mathrm{H}_{2}$ and $\mathrm{CH}_{3} \mathrm{OH}$ peaks.

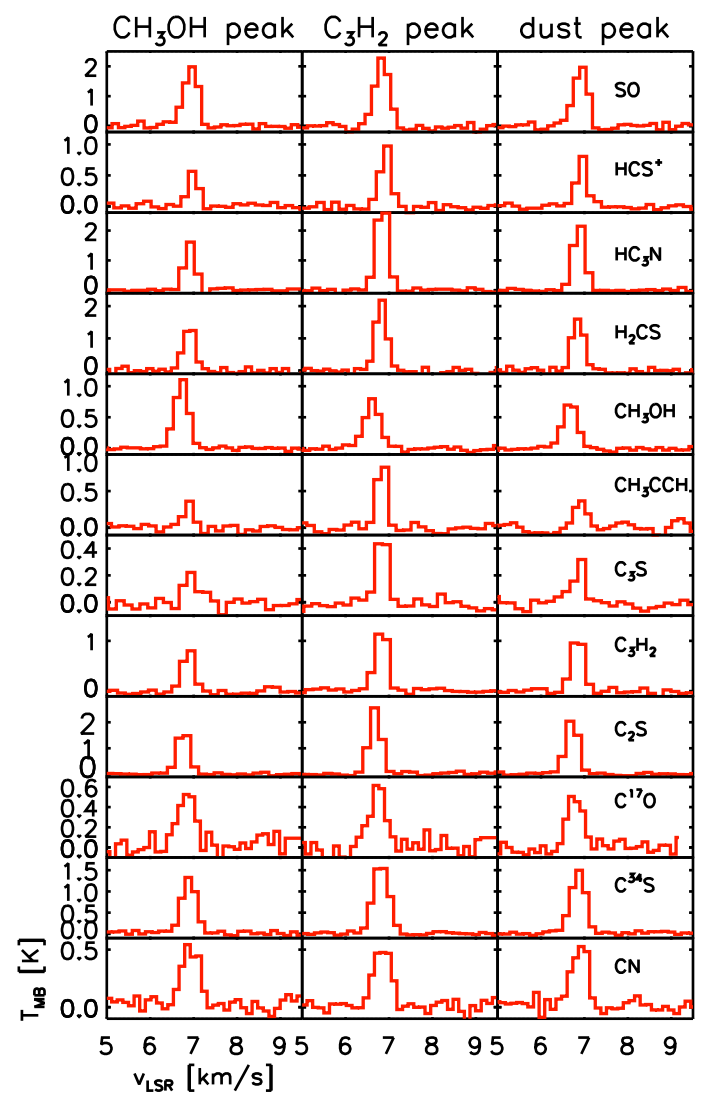

Fig. 3. Line profiles of selected molecules observed toward $\mathrm{CH}_{3} \mathrm{OH}$ peak, $c-\mathrm{C}_{3} \mathrm{H}_{2}$ peak, and Herschel dust peak. The transitions used for each molecule are marked in Table A.1.

The third and fourth PCs account for only 2.9 and $2.4 \%$ of the correlation and are likely to be dominated by noise. Figure 7 shows the contribution of each molecule to the four PCs, and Fig. 8 shows correlation wheels where the coordinates for each molecule are given by their correlation coefficients to each PC, obtained by performing the PCA on the standardized data.

The PCA results for L1521E and those presented by Spezzano et al. (2017) for L1544 are similar, as seen in Fig. 6 from this paper and in Fig. 2 from Spezzano et al. (2017). The first PC follows the distribution of the continuum in both cases, however, it represents $78.0 \%$ of the correlation in the dataset for L1521E, and only $67 \%$ for L1544. The second PC shows the methanol and $c-\mathrm{C}_{3} \mathrm{H}_{2}$ peaks in case of both sources, but it represents a higher correlation (12.2\%) for L1544 than for L1521E $(8.0 \%)$. The differences between the results for the two cores may be related to the fact that while 28 different molecular line maps were included in the PCA for L1544, only 24 species were available with a sufficient S/N level toward L1521E.

\subsection{Molecular column densities}

We derived column densities for the observed species toward the Herschel dust peak in an optically thin approximation, assuming a single excitation temperature and negligible background radiation. We measured the excitation temperature by fitting the hyperfine structure of the $\mathrm{N}_{2} \mathrm{H}^{+}(1-0)$ line toward the Herschel dust peak, using Gildas/CLASS. The excitation temperature given by the fit is $\sim 4.5 \mathrm{~K}$. We used this excitation temperature to derive the column density of all the species, except for $\mathrm{C}^{17} \mathrm{O}$. Due to its low critical density $\left(\sim 2 \times 10^{3} \mathrm{~cm}^{-3}\right), \mathrm{C}^{17} \mathrm{O}$ is supposed 


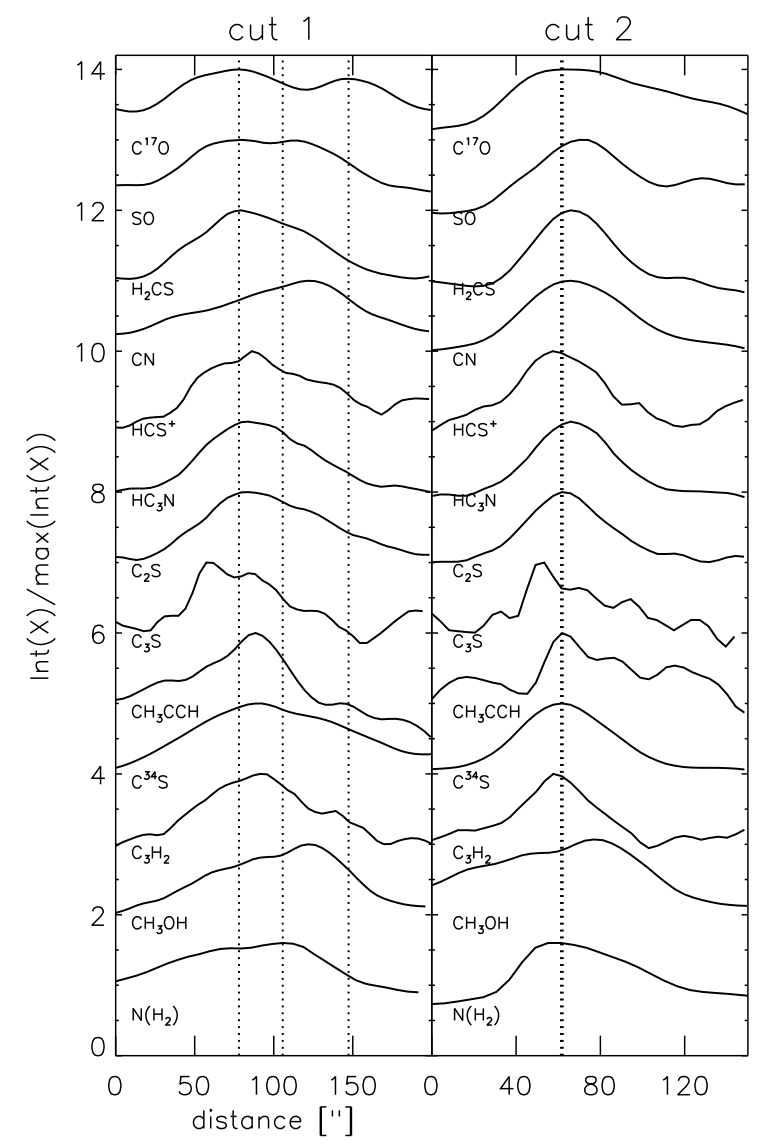

Fig. 4. Left: intensity cuts of selected molecules extracted along line crossing the two $\mathrm{C}^{17} \mathrm{O}$ peaks (close to $c-\mathrm{C}_{3} \mathrm{H}_{2}$ and $\mathrm{CH}_{3} \mathrm{OH}$ peaks) for various species (cut 1 in Fig. 1). Right: intensity cuts extracted along a line perpendicular to other cut and crosses Herschel dust peak (cut 2 in Fig. 1). The intensity values ( $y$-axis) are normalized to the maximum value for each species, and a constant has been added to compare the normalized intensity distributions of the different species. The vertical dotted lines show the locations of the $\mathrm{C}^{17} \mathrm{O}$ and $N\left(\mathrm{H}_{2}\right)$ peaks.

to be thermalized, therefore we assumed the excitation temperature for $\mathrm{C}^{17} \mathrm{O}$ to be $10 \mathrm{~K}$, which is close to the dust temperature of $9.8 \mathrm{~K}$ derived by Makiwa et al. (2016). The measured integrated intensities of the lines $\left(\int T_{\mathrm{MB}} \mathrm{d} V\right)$ can be converted to the column densities of the molecules $\left(N_{\text {tot }}\right)$ in optically thin approximation using:

$N_{\mathrm{tot}}=\frac{8 \pi k v^{2}}{h c^{3}} \frac{Q\left(T_{\mathrm{ex}}\right)}{g_{\mathrm{u}} A_{\mathrm{ul}}} e^{E_{\mathrm{u}} / k T_{\mathrm{ex}}} \int T_{\mathrm{mb}} \mathrm{d} v$,

where $g_{\mathrm{u}}$ is the statistical weight of the upper level, $Q\left(T_{\mathrm{ex}}\right)$ is the partition function for $T_{\mathrm{ex}}, E_{\mathrm{u}}$ is the upper level energy, $v$ is the frequency of the used transition, and $A_{\mathrm{ul}}$ is the spontaneous decay rate. Eq. (1) also assumes a beam filling factor of 1, which means that the source emission is assumed to be more extended than the beam size. The column densities of the species are summarized in Table 1. The transitions used to calculate column densities are marked with an asterisk in Table A.1. The error of the column densities is dominated by calibration error and error given by the uncertainty of the assumed excitation temperature.

\subsection{CO depletion}

As already seen in Fig. 5, the $\mathrm{C}^{17} \mathrm{O}$ line intensities decrease around the Herschel dust peak, which suggests that there is some
Table 1. Molecular column densities derived toward the dust peaks of L1521E and L1544.

\begin{tabular}{|c|c|c|}
\hline \multirow[t]{2}{*}{ Molecule } & \multicolumn{2}{|c|}{ Column density $\left(\mathrm{cm}^{-2}\right)$} \\
\hline & L1521E & L1544 \\
\hline $\mathrm{O}$ & $(1.6 \pm 0.5) \times 10^{13}$ & $(1.5 \pm 0.5) \times 10^{13}$ \\
\hline & $(1.6 \pm 0.5) \times 10^{12}$ & $(2.5 \pm 0.8) \times 10^{11}$ \\
\hline OCS & $(2.3 \pm 0.7) \times 10^{13}$ & $(8.9 \pm 2.7) \times 10^{12}$ \\
\hline $\mathrm{C}_{2} \mathrm{~S}$ & $(1.4 \pm 0.4) \times 10^{13}$ & $(4.8 \pm 1.4) \times 10^{12}$ \\
\hline $\mathrm{C}_{3} \mathrm{~S}$ & $(8.2 \pm 2.5) \times 10^{13}$ & $(5.7 \pm 1.7) \times 10^{12}$ \\
\hline $\mathrm{C}^{33} \mathrm{~S}$ & $(1.4 \pm 0.4) \times 10^{12}$ & $(2.3 \pm 0.7) \times 10^{11}$ \\
\hline $\mathrm{C}^{34} \mathrm{~S}$ & $(2.9 \pm 0.9) \times 10^{12}$ & $(6.1 \pm 1.8) \times 10^{11}$ \\
\hline $\mathrm{CC}^{34} \mathrm{~S}$ & $(8.3 \pm 2.5) \times 10^{11}$ & - \\
\hline $\mathrm{HCS}^{+}$ & $(1.1 \pm 0.3) \times 10^{12}$ & $(4.4 \pm 1.3) \times 10^{11}$ \\
\hline $\mathrm{H}_{2} \mathrm{CS}$ & $(1.8 \pm 0.5) \times 10^{13}$ & $(6.0 \pm 1.8) \times 10^{12}$ \\
\hline $\mathrm{C}^{17} \mathrm{O}$ & $(1.2 \pm 0.4) \times 10^{14}$ & - \\
\hline $\mathrm{HC}_{3} \mathrm{~N}$ & $(8.4 \pm 2.5) \times 10^{12}$ & $(1.1 \pm 0.3) \times 10^{13}$ \\
\hline$c-\mathrm{C}_{3} \mathrm{H}_{2}$ & $(1.3 \pm 0$ & $(4.3 \pm 1.3) \times 10^{12}$ \\
\hline $\mathrm{C}_{4} \mathrm{H}$ & $(1.6 \pm 0.5) \times 10^{14}$ & $(2.0 \pm 0.6) \times 10^{14}$ \\
\hline $\mathrm{HN}^{13} \mathrm{C}$ & $(6.0 \pm 1.8) \times 10^{11}$ & $(2.0 \pm 0.6)$ \\
\hline $\mathrm{CH}_{3} \mathrm{OH}\left(E_{2}\right)$ & $(1.9 \pm 0.6) \times 10^{13}$ & $(2.2 \pm 0.7) \times 10^{13}$ \\
\hline $\mathrm{CH}_{3} \mathrm{OH}\left(A^{+}\right)$ & $(5.7 \pm 1.7) \times 10^{12}$ & $(7.3 \pm 2.2) \times 10^{12}$ \\
\hline $\mathrm{CH}_{3} \mathrm{CCH}$ & $(2.1 \pm 0.6) \times 10^{13}$ & $(4.9 \pm 1.5) \times 10^{13}$ \\
\hline $\mathrm{CH}_{3} \mathrm{CHO}$ & $(8.3 \pm 2.5) \times 10^{10}$ & $(6.2 \pm 1.9) \times 10^{10}$ \\
\hline$l-\mathrm{C}_{4} \mathrm{H}_{2}$ & $(4.1 \pm 1.2) \times 10^{11}$ & - \\
\hline $\mathrm{H}_{2} \mathrm{CCO}$ & $(1.4 \pm 0.4) \times 10^{12}$ & - \\
\hline $\mathrm{CH}_{3} \mathrm{CN}$ & $(4.8 \pm 1.4) \times 10^{11}$ & $(6.1 \pm 1.8) \times 10^{11}$ \\
\hline $\mathrm{HC}^{18} \mathrm{O}^{+}$ & $(6.7 \pm 2.0) \times 10^{10}$ & $(8.3 \pm 2.5) \times 10^{10}$ \\
\hline $\mathrm{H}^{13} \mathrm{CN}$ & $(1.6 \pm 0.5) \times 10^{12}$ & $(2.6 \pm 0.8) \times 10^{12}$ \\
\hline $\mathrm{HCO}$ & $(7.0 \pm 2.1) \times 10^{11}$ & $(5.0 \pm 1.5) \times 10^{11}$ \\
\hline $\mathrm{HCN}$ & $(2.4 \pm 0.7) \times 10^{12}$ & $(1.3 \pm 0.4) \times 10^{12}$ \\
\hline $\mathrm{HCO}^{+}$ & $(1.3 \pm 0.4) \times 10^{12}$ & $(3.2 \pm 1.0) \times 10^{11}$ \\
\hline $\mathrm{N}_{2} \mathrm{H}^{+}$ & $(2.5 \pm 0.8) \times 10^{13}$ & $(2.0 \pm 0.6) \times 10^{14}$ \\
\hline $\mathrm{HNCO}$ & $(9.3 \pm 2.8) \times 10^{12}$ & $(1.6 \pm 0.5) \times 10^{13}$ \\
\hline $\mathrm{C}_{2} \mathrm{H}$ & $(7.6 \pm 2.3) \times 10^{13}$ & $(9.5 \pm 2.9) \times 10^{13}$ \\
\hline $\mathrm{CN}$ & $(1.2 \pm 0.4) \times 10^{13}$ & - \\
\hline
\end{tabular}

Notes. The column densities toward L1544 are based on data from the ASAI (Lefloch et al. 2018). The assumed excitation temperature was $10 \mathrm{~K}$ for $\mathrm{CO}$ and $4.5 \mathrm{~K}$ for the other species for L1521E, and $5 \mathrm{~K}$ for L1544. The used transitions are shown in Table A.1 with asterisk, except for $\mathrm{C}_{3} \mathrm{~S}(92488.5 \mathrm{MHz}), \mathrm{CH}_{3} \mathrm{CHO}(95947.4 \mathrm{MHz})$, and $\mathrm{H}_{2} \mathrm{CCO}$ (101981.4 MHz), to be consistent between the two sources. The SO column density is based on the ${ }^{34} \mathrm{SO}$ column density and an isotopic ratio of 22 (Wilson \& Rood 1994).

$\mathrm{CO}$ depletion. To calculate the $\mathrm{CO}$ depletion factors we used the observed $\mathrm{C}^{17} \mathrm{O}$ map and the $N\left(\mathrm{H}_{2}\right)$ map derived from the Herschel/SPIRE data. We use the $J=1-0, F=5 / 2-5 / 2$ transition of $\mathrm{C}^{17} \mathrm{O}$ at $112.36 \mathrm{GHz}$ to derive the $\mathrm{C}^{17} \mathrm{O}$ column density in optically thin LTE approximation assuming an excitation temperature of $10 \mathrm{~K}$ (Tafalla \& Santiago 2004). For this analysis we smoothed the $\mathrm{C}^{17} \mathrm{O}$ map from its original spatial resolution $\left(\sim 30^{\prime \prime}\right)$ to the resolution of the $N\left(\mathrm{H}_{2}\right)$ map from the Herschel/SPIRE data $\left(\sim 40^{\prime \prime}\right)$. We used a ${ }^{16} \mathrm{O} /{ }^{17} \mathrm{O}$ abundance ratio of 2044 (Penzias 1981; Wilson \& Rood 1994) and a canonical CO abundance of $8.5 \times 10^{-5}$ (Frerking et al. 1982).

To derive $N\left(\mathrm{H}_{2}\right)$ from the Herschel/SPIRE fluxes we used a dust emissivity index of $\beta=1.5$. The value of $\beta=1.5$ gives a good comparison with $\mathrm{L} 1544$ as it was used to derive $N\left(\mathrm{H}_{2}\right)$ for that source (Spezzano et al. 2016). Other studies, such as Kirk et al. (2007) assumed a value of $\beta=2.0$ for L1521E. A $\beta=2.6 \pm 0.9$, 

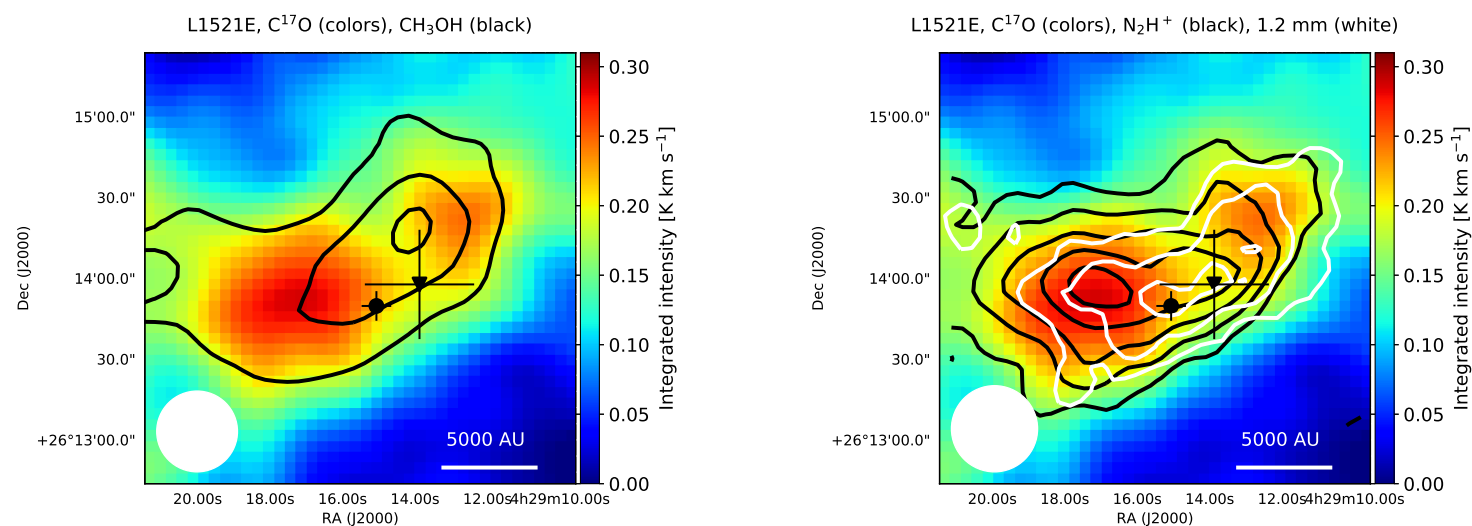

Fig. 5. Structure of L1521E. Left: integrated intensity of $\mathrm{CH}_{3} \mathrm{OH}$ (black contours) overplotted on integrated intensity of $\mathrm{C}^{17} \mathrm{O}$. The black dot shows the peak of the $1.2 \mathrm{~mm}$ emission. The black triangle shows the peak of the $\mathrm{H}_{2}$ column density derived from the Herschel/SPIRE data. The $\mathrm{CH}_{3} \mathrm{OH}$ contour levels start from $6 \sigma \mathrm{rms}$ in steps of $6 \sigma \mathrm{rms}$, with a $3 \sigma \mathrm{rms}$ level of $0.07 \mathrm{~K} \mathrm{~km} \mathrm{~s}^{-1}$. Right: integrated intensity of $\mathrm{N}_{2} \mathrm{H}^{+}$(black contours) and distribution of $1.2 \mathrm{~mm}$ emission (white contours) overplotted on integrated intensity of $\mathrm{C}^{17} \mathrm{O}$. The black dot shows the peak of the $1.2 \mathrm{~mm}$ emission. The black triangle shows the peak of the $\mathrm{H}_{2}$ column density derived from the Herschel/SPIRE data. The $\mathrm{N}_{2} \mathrm{H}^{+}$contour levels start from $6 \sigma \mathrm{rms}$ in steps of $4 \sigma \mathrm{rms}$, with $3 \sigma \mathrm{rms}$ level of $0.08 \mathrm{~K} \mathrm{~km} \mathrm{~s}^{-1}$. For the $1.2 \mathrm{~mm}$ data the contours start from $6 \sigma \mathrm{rms}$ in steps of $3 \sigma \mathrm{rms}$ with a $3 \sigma \mathrm{rms}$ level of $5.38 \mathrm{mJy}$.
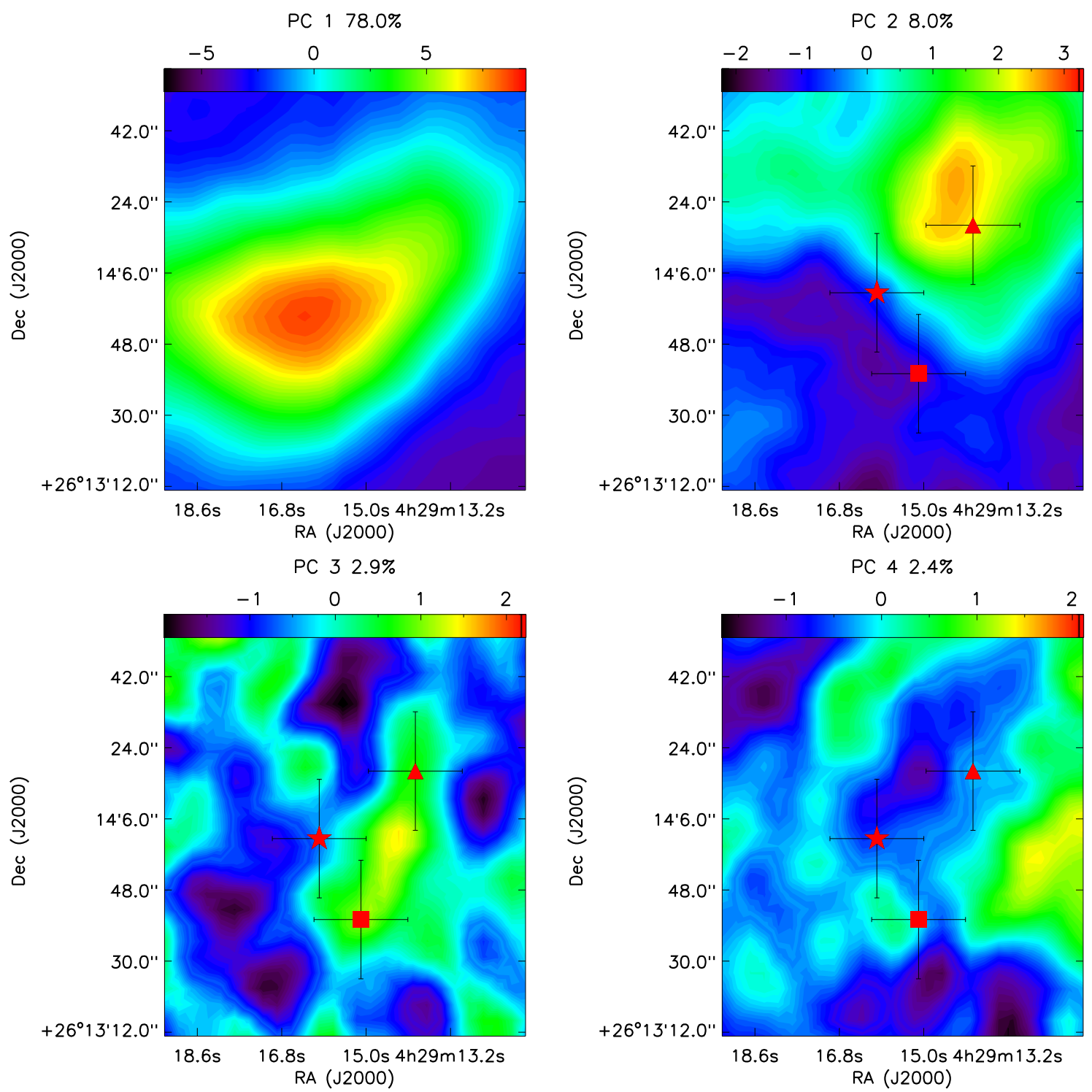

Fig. 6. Maps of first four principal components obtained by performing PCA. The percentages represent the amount of correlation that can be reproduced by the single principal component. The red triangle represents the $\mathrm{CH}_{3} \mathrm{OH}$ peak, the asterisk is the $c-\mathrm{C}_{3} \mathrm{H}_{2}$ peak, and the square the is HNCO peak. 

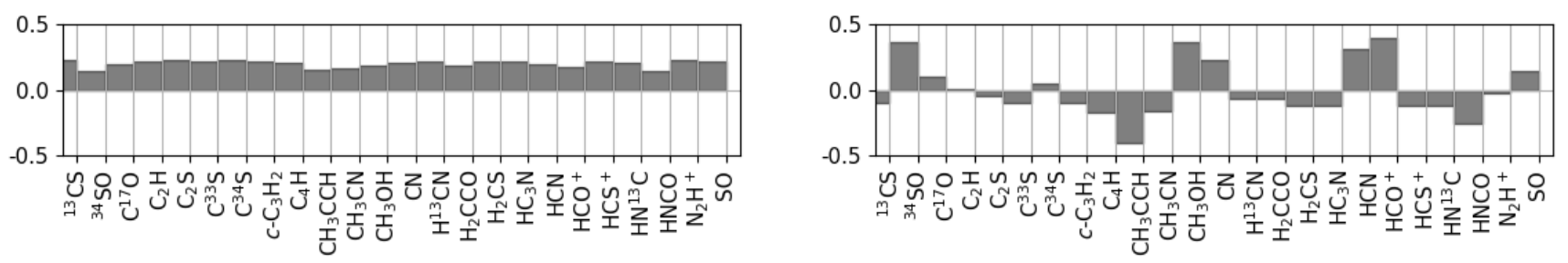

Fig. 7. Contribution of each transition to first two PCs, obtained by performing PCA on the standardized data.

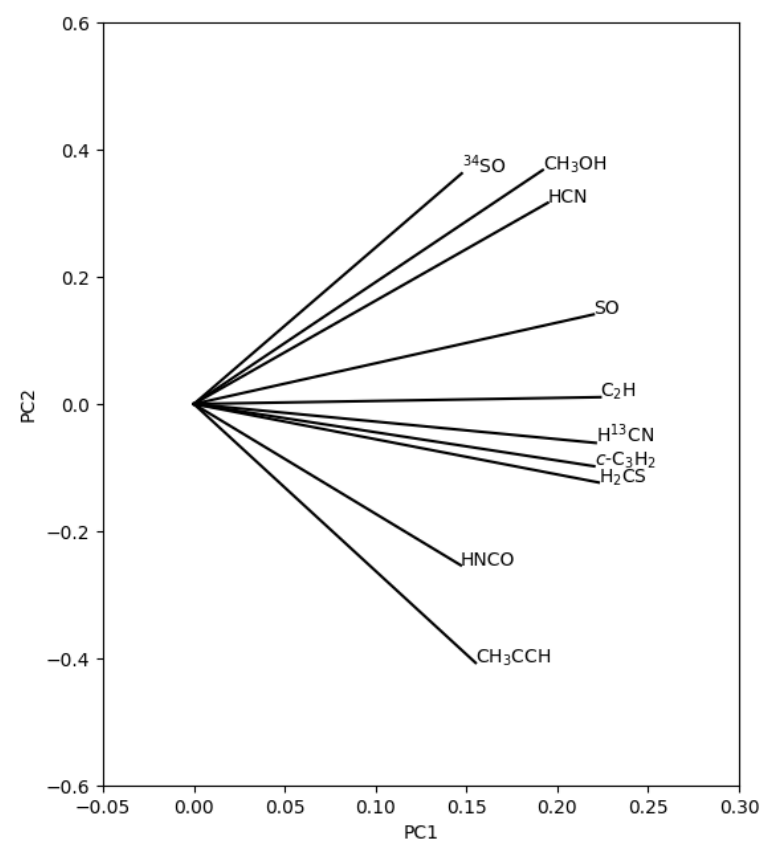

Fig. 8. Correlation wheels where the coordinates for each molecule are their contribution to each PC, obtained by performing the PCA on the standardised data.

which was derived from Herschel/SPIRE data by Makiwa et al. (2016) is also consistent with this value.

The calculation for $\beta=1.5$ results in a $\mathrm{CO}$ depletion factor of $4.3 \pm 1.6$ toward the Herschel dust peak. The derived depletion factors are shown in Fig. 9, with the methanol integrated intensity contours overplotted. Even though the depletion factors do not peak at the same position as the methanol intensities, the depletion factors increase with increasing methanol abundance, which was expected as $\mathrm{CH}_{3} \mathrm{OH}$ is produced on the surface of dust grains by $\mathrm{CO}$ hydrogenation (Watanabe \& Kouchi 2002) and then released to the gas phase via reactive desorption (Vasyunin et al. 2017).

We also used the $1.2 \mathrm{~mm}$ data to derive $N\left(\mathrm{H}_{2}\right)$ from a different method described in Crapsi et al. (2005). We smoothed the $1.2 \mathrm{~mm}$ map to the resolution of the IRAM-30 m data $\left(\sim 30^{\prime \prime}\right)$. For the dust opacity per unit mass we used a value of $\kappa_{1.2 \mathrm{~mm}}=$ $0.005 \mathrm{~cm}^{2} \mathrm{~g}^{-1}$. This value was chosen following Crapsi et al. (2005), who derived depletion factors for a number of starless cores. Originally, André et al. (1996) chose this value based on the recommendation of Henning et al. (1995) and Preibisch et al. (1993) for clouds at intermediate densities $\left(\sim 10^{5} \mathrm{~cm}^{-3}\right)$ and gasto-dust ratios of 100 . The $\mathrm{CO}$ depletion factors resulting from this method are similar to the values derived using $N\left(\mathrm{H}_{2}\right)$ values from the Herschel data, with a value of $3.9 \pm 1.4$ toward the Herschel dust peak. The large depletion factors based on the $1.2 \mathrm{~mm}$ data in the southern part of the map in Fig. 9 are probably an artifact due to the filtering of the extended cloud structure by MAMBO-2. The $\mathrm{S} / \mathrm{N}$ in these parts of the map is below three.

Crapsi et al. (2005) calculated depletion factors for several starless cores that are in the range between $3.4 \pm 2.1$ and $15.5 \pm 3.7$, including the value $14 \pm 2.2$ for L 1544 , which is about three times the value derived for $\operatorname{L1521E}(4.3 \pm 1.6)$ when assuming $\beta=1.5$ for the $\mathrm{H}_{2}$ column density. Based on the comparison of $\mathrm{C}^{18} \mathrm{O}$ observations of L1521E to chemical models, Tafalla \& Santiago (2004) concluded that CO depletion is not present in L1521E, given that the data are consistent with a constant abundance profile. Similarly, Ford \& Shirley (2011) found little or no $\mathrm{C}^{18} \mathrm{O}$ depletion toward L1521E by the comparison of observations and chemical models. Tafalla \& Santiago (2004) found some differences between the distribution of the $\mathrm{C}^{18} \mathrm{O}$ and continuum emission outside the core central region (which is also seen in Fig. 1 from Ford \& Shirley 2011), but attributed them to the low sensitivity of the data at those positions, and not to $\mathrm{CO}$ depletion. The depletion factor of $4.3 \pm 1.6$ found using the data presented here and the spatial distribution of $\mathrm{C}^{17} \mathrm{O}(1-0)$ suggest that some $\mathrm{CO}$ depletion is present in L1521E near the dust peak.

\subsection{Comparison to models}

To understand the measured column densities, we preformed chemical modeling using the 1D code described in Vasyunin et al. (2017). The model represents the core with a spherically symmetrical structure. We used the density structure reported by Tafalla $\&$ Santiago (2004) and a constant temperature profile at $T=10 \mathrm{~K}$, also used by Tafalla \& Santiago (2004). In the model we calculated time-dependent fractional abundances and column densities of species. Then the calculated column densities are convolved with a $26^{\prime \prime}$ Gaussian beam, to be comparable to those measured with the IRAM-30 m. In the model, only the main isotopologs of the species are available, therefore, we used isotopic ratios based on Wilson \& Rood (1994) to compare the modeled and observed column densities. We ran several models with different initial abundances and compared the resulting column densities to those obtained from the observations, to find a model that fits the most observed column densities within an order of magnitude. The best fit model uses "low metals" (EA1) initial abundances from Wakelam \& Herbst (2008), except for the fractional abundance of $\mathrm{S}^{+}$, which needs to be increased to $10^{-6}$ instead of $8 \times 10^{-8}$ to fit the observations. The model was run in two stages. First, we ran the evolution of a diffuse cloud for $10^{6} \mathrm{yr}$. Then, we took the final abundances of species from the first stage as the initial chemical composition for the next stage, and ran the evolution of L1521E itself. The best fit column densities from the models are shown in Table 2 and in Fig. 10 as a function of radius. This model is able to reproduce most measured column densities, especially those of the sulfur-bearing molecules except for SO. This supports the result that significant sulfur depletion takes place during the evolution 

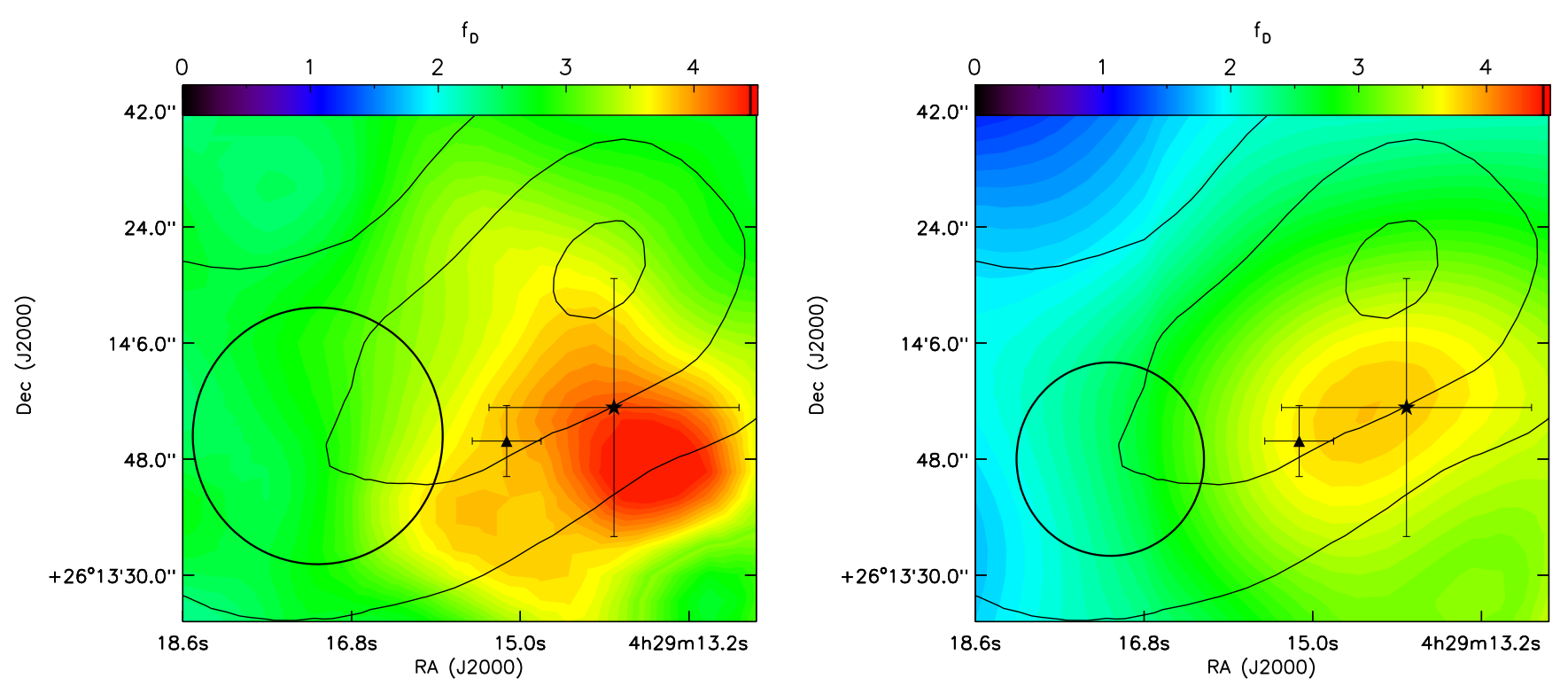

Fig. 9. Map of $\mathrm{CO}$ depletion factors toward L1521E. These were calculated using $\mathrm{H}_{2}$ column densities based on the Herschel/SPIRE data with $\beta=1.5$ (left panel) and based on the $1.2 \mathrm{~mm}$ map (right panel), the derived $\mathrm{C}^{17} \mathrm{O}$ abundances, and a reference value of $\mathrm{X}\left(\mathrm{C}^{17} \mathrm{O}\right)=5.4 \times 10^{-8}$. The contours show the spatial distribution of methanol and start from $6 \sigma \mathrm{rms}$ noise level in steps of $6 \sigma \mathrm{rms}$. The triangle shows the peak of the $1.2 \mathrm{~mm}$ emission and the asterisk symbol shows the Herschel dust peak. The depletion factor toward the Herschel dust peak is about 4.3 based on the Herschel $N\left(\mathrm{H}_{2}\right)$ values and about 3.9 based on the $N\left(\mathrm{H}_{2}\right)$ values calculated from the $1.2 \mathrm{~mm}$ data. The black circles show the beam sizes that were used to calculate the depletion factors.

Table 2. Best fit column densities derived for L1521E with chemical code described in Vasyunin et al. (2017) and agreement with measured values given in Fig. 11.

\begin{tabular}{|c|c|c|}
\hline Molecule & Column density $\left(\mathrm{cm}^{-2}\right)$ & Agree(+)/disagree (-) \\
\hline SO & $3.5 \times 10^{15}$ & - \\
\hline $\mathrm{CS}$ & $1.8 \times 10^{14}$ & + \\
\hline OCS & $1.0 \times 10^{13}$ & + \\
\hline $\mathrm{C}_{2} \mathrm{~S}$ & $8.5 \times 10^{13}$ & + \\
\hline $\mathrm{C}_{3} \mathrm{~S}$ & $2.7 \times 10^{13}$ & + \\
\hline $\mathrm{HCS}^{+}$ & $2.7 \times 10^{11}$ & + \\
\hline $\mathrm{H}_{2} \mathrm{CS}$ & $8.2 \times 10^{13}$ & + \\
\hline $\mathrm{CO}$ & $4.9 \times 10^{17}$ & + \\
\hline $\mathrm{HC}_{3} \mathrm{~N}$ & $1.2 \times 10^{12}$ & + \\
\hline$c-\mathrm{C}_{3} \mathrm{H}_{2}$ & $9.5 \times 10^{13}$ & - \\
\hline $\mathrm{C}_{4} \mathrm{H}$ & $3.2 \times 10^{13}$ & + \\
\hline $\mathrm{HNC}$ & $3.1 \times 10^{13}$ & + \\
\hline $\mathrm{CH}_{3} \mathrm{OH}$ & $5.3 \times 10^{13}$ & + \\
\hline $\mathrm{CH}_{3} \mathrm{CCH}$ & $2.4 \times 10^{11}$ & - \\
\hline $\mathrm{CH}_{3} \mathrm{CHO}$ & $1.3 \times 10^{11}$ & + \\
\hline$l-\mathrm{C}_{4} \mathrm{H}_{2}$ & $4.7 \times 10^{13}$ & - \\
\hline $\mathrm{H}_{2} \mathrm{CCO}$ & $4.2 \times 10^{12}$ & + \\
\hline $\mathrm{CH}_{3} \mathrm{CN}$ & $1.3 \times 10^{11}$ & + \\
\hline $\mathrm{HCO}^{+}$ & $1.7 \times 10^{13}$ & + \\
\hline $\mathrm{HCN}$ & $3.5 \times 10^{13}$ & + \\
\hline $\mathrm{HCO}$ & $3.9 \times 10^{12}$ & + \\
\hline $\mathrm{C}_{2} \mathrm{H}$ & $5.3 \times 10^{13}$ & + \\
\hline HNCO & $1.2 \times 10^{13}$ & + \\
\hline $\mathrm{N}_{2} \mathrm{H}^{+}$ & $4.8 \times 10^{12}$ & + \\
\hline $\mathrm{CN}$ & $6.2 \times 10^{13}$ & + \\
\hline
\end{tabular}

of dense cores. The best fit model suggests a chemical age of $\sim 1.7 \times 10^{5}$ years, which is close to what was found by Tafalla \& Santiago (2004). For this age estimate $t=0$ is the start of the second stage of the model run mentioned above, when the adopted 1D physical structure of the core has been formed. Since our physical model does not evolve with time, the obtained age is rather a timescale of the relaxation for chemical evolution of the model core up to the observed chemical composition rather than an estimate of the physical age of the real core. The $\mathrm{CO}$ depletion factor at the moment of best agreement towards the center of L1521E in the model is $\sim 3$, which is close to the value of $4.3 \pm 1.6$ derived from the observations.

\section{Discussion}

We analyzed IRAM-30 m observations of various molecules toward the starless core L1521E. We found that, similarly to L1544, $\mathrm{CH}_{3} \mathrm{OH}$ and $c-\mathrm{C}_{3} \mathrm{H}_{2}$ peak at two different positions, but the distance between the two peaks is smaller and similar to the size of the $\mathrm{CO}$ depletion zone (in both cases). Most observed species peak at the $c-\mathrm{C}_{3} \mathrm{H}_{2}$ peak. The $\mathrm{C}^{17} \mathrm{O}$ column densities calculated toward L1521E suggest that $\mathrm{CO}$ is depleted toward the Herschel dust peak. The depletion factor based on the $\mathrm{C}^{17} \mathrm{O}$ data and $N\left(\mathrm{H}_{2}\right)$ calculated from Herschel/SPIRE data was found to be $4.3 \pm 1.6$ and the depletion factor based on the $1.2 \mathrm{~mm}$ emission was found to be $3.9 \pm 1.4$.

We compared the column density ratios of the species measured toward the dust peak of L1521E with those measured toward the dust peak of L1544. To estimate column densities toward L1544 we used the spectrum measured toward L1544 in the 81-106 GHz range as part of the ASAI (Lefloch et al. 2018). The column densities of the species toward L1544 were derived with the same method as for L1521E described in Sect. 4.3, using the same transitions of the species toward both cores. We assumed an excitation temperature of $5 \mathrm{~K}$ which was derived by Crapsi et al. (2005) by fitting the $\mathrm{N}_{2} \mathrm{H}^{+}(1-0)$ hyperfine structure. Since the $\mathrm{H}_{2}$ column densities reported toward L1544 define a significant range, from $2.8 \times 10^{22} \mathrm{~cm}^{-2}$ (Spezzano et al. 2016) derived from Herschel/SPIRE data to $(9.4 \pm 1.6) \times 10^{22} \mathrm{~cm}^{-2}$ 

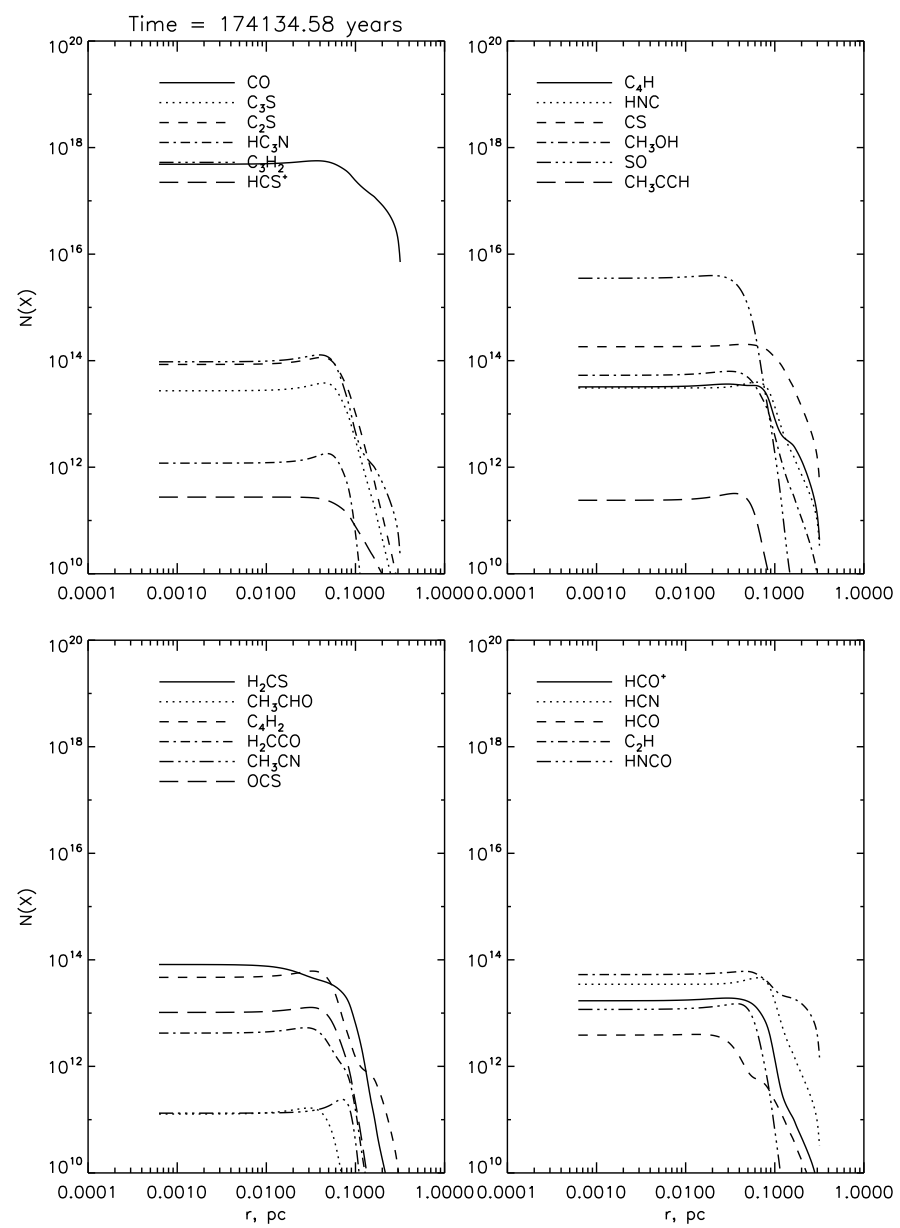

Fig. 10. Column densities of different species as function of model predicted radius.

derived from the $1.2 \mathrm{~mm}$ continuum (Crapsi et al. 2005), we compared column density ratios toward the two cores. It is important to note that the column densities were derived from data measured with similar beam sizes. The column density ratios with the reference column density of $\mathrm{CH}_{3} \mathrm{OH}\left(\mathrm{A}^{+}\right)$toward the two cores are shown in Fig. 11. One of the main differences between the column density ratios calculated toward the two sources is seen for the sulfur-bearing species with carbon, such as $\mathrm{C}_{2} \mathrm{~S}, \mathrm{HCS}^{+}, \mathrm{C}^{34} \mathrm{~S}, \mathrm{C}^{33} \mathrm{~S}$, and $\mathrm{HCS}^{+}$, which have higher abundances toward L1521E than toward L1544. These Cand S-bearing molecules were found to follow the $c-\mathrm{C}_{3} \mathrm{H}_{2}$ peak in L1544 (Spezzano et al. 2017), meaning toward L1544 they appear to trace chemically young gas, probably affected by the interstellar radiation field. The relatively higher abundance of these molecules toward L1521E may indicate that sulfur depletion continues to take place during the dynamical evolution of dense cores, as also recently found by Laas \& Caselli (2019), therefore, organic S-bearing molecules can be considered good tracers of chemically young dense cores. Among the S-bearing species studied here, SO was also found to trace cores in an early evolutionary state by other studies, such as Hacar et al. (2013), who found extended SO emission toward the L1495/B213 complex in Taurus. They interpreted SO to trace gas in regions of early evolutionary state as it was found to be bright in regions with bright $\mathrm{C}^{18} \mathrm{O}$ emission and intense dust millimeter continuum together with little $\mathrm{N}_{2} \mathrm{H}^{+}$emission. Tafalla et al. (2006) studied the abundances of molecules toward the L1498 and
L1517B starless cores in Taurus. Based on the comparison of observations to radiative transfer models they found $\mathrm{SO}$ and $\mathrm{C}_{2} \mathrm{~S}$ to be good tracers of molecular depletion.

The $\mathrm{CH}_{3} \mathrm{OH}\left(E_{2}\right)$ relative abundances are similar in the two cores. The fact that the $\mathrm{N}_{2} \mathrm{H}^{+}$abundance toward L1521E is higher than the abundance toward L1544 confirms that L1521E is indeed in an earlier stage of core evolution, due to the relatively long formation route of $\mathrm{N}_{2} \mathrm{H}^{+}$starting from atomic nitrogen (e.g. Hily-Blant et al. 2010).

In addition to the abundances, the spatial distribution of species helps us to better understand the difference between the two cores. Spezzano et al. (2017) found four different molecular peaks toward L1544: dust peak, $c-\mathrm{C}_{3} \mathrm{H}_{2}$ peak, methanol peak, and $\mathrm{HNCO}$ peak. These peaks are also present toward L1521E, but they are closer together, compared to the more dynamically evolved L1544 pre-stellar core. $\mathrm{N}_{2} \mathrm{H}^{+}$and $\mathrm{CN}$ peak close to the dust peak toward L1521E, and similarly $\mathrm{N}_{2} \mathrm{H}^{+}$and ${ }^{13} \mathrm{CN}$ also peak close to the dust peak toward L1544 (Spezzano et al. 2017). Not many species were found to peak toward the methanol peak in L1521E. SO, which peaks toward the methanol peak in L1544 shows two peaks in L1521E, one toward the $c-\mathrm{C}_{3} \mathrm{H}_{2}$, and one close to the $\mathrm{CH}_{3} \mathrm{OH}$ peak. Spezzano et al. (2017) found that Oand $\mathrm{S}$-bearing molecules follow the methanol distribution toward L1544, and therefore trace more chemically evolved gas or gas that is more protected from the interstellar radiation field (see also Swade 1989).

Most species (mostly carbon chain molecules) peak toward the $c-\mathrm{C}_{3} \mathrm{H}_{2}$ peak in both cores. One difference is $\mathrm{CH}_{3} \mathrm{CCH}$, which peaks toward the $c-\mathrm{C}_{3} \mathrm{H}_{2}$ peak in $\mathrm{L} 1521 \mathrm{E}$, and toward the HNCO peak in L1544.

As seen in Sect. $4.1 c-\mathrm{C}_{3} \mathrm{H}_{2}$ and $\mathrm{CH}_{3} \mathrm{OH}$ peak at different positions, similar to what was observed toward L1544 (Spezzano et al. 2016). However, the interpretation of the different spatial distributions is different for L1521E than for L1544. As it was shown by Spezzano et al. (2016) for L1544 (and unlike $\mathrm{CH}_{3} \mathrm{OH}$ as in Fig. 12), $c-\mathrm{C}_{3} \mathrm{H}_{2}$ probably originates in a layer-like structure as its intensities do not correlate with the $\mathrm{H}_{2}$ column densities, but rather show a flat distribution, tracing the external core envelope. However in the case of L1521E, even though they peak at different positions toward the core, $c-\mathrm{C}_{3} \mathrm{H}_{2}$ and $\mathrm{CH}_{3} \mathrm{OH}$ both show a correlation with the $\mathrm{H}_{2}$ column densities, which can be understood if the central density of the L1521E core is not high enough for catastrophic freeze-out to take place.

Overall, the chemical segregation in L1521E is not as developed as in L1544, which is probably related to the earlier evolutionary state of L1521E, and the fact that L1521E has not developed a high density central region, where freeze-out of $\mathrm{C}$ and O-bearing bearing molecules dominates.

\section{Summary}

We presented observations toward the starless core L1521E carried out with the IRAM-30 m telescope. The main results can be summarized as follows:

- The lower limit on the CO depletion factor toward the dust peak of L1521E was found to be $4.3 \pm 1.6$, which is about one third of the value measured toward the more evolved L1544 core.

- $c-\mathrm{C}_{3} \mathrm{H}_{2}$ and $\mathrm{CH}_{3} \mathrm{OH}$ peak at different positions in both L1521E and L1544 (based on Spezzano et al. 2016), but the origin of the distributions toward the two cores is different, as toward L1521E both molecules show an increasing trend with increasing $N\left(\mathrm{H}_{2}\right)$ while toward L1544 only the 


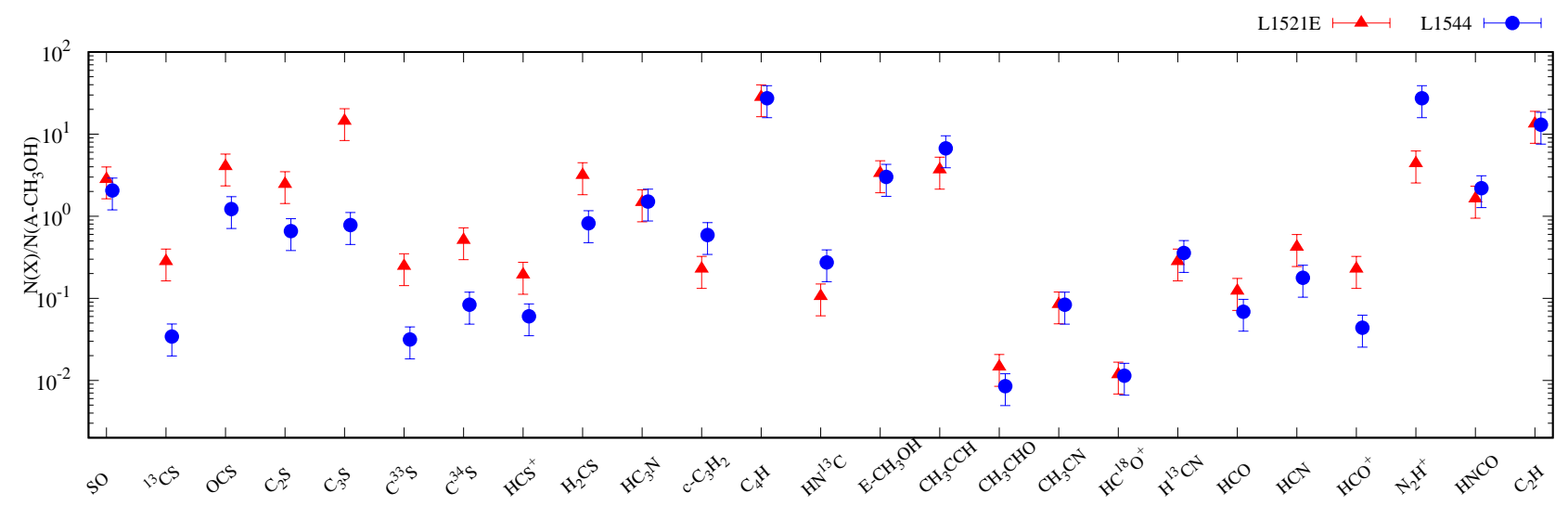

Fig. 11. Comparison of relative abundances of species observed toward dust peak of both L1521E and L1544. As explained in Sect. 4.1, the abundances were derived using the column densities for an excitation temperature of $4.5 \mathrm{~K}$ for L1521E and $5 \mathrm{~K}$ for L1544.

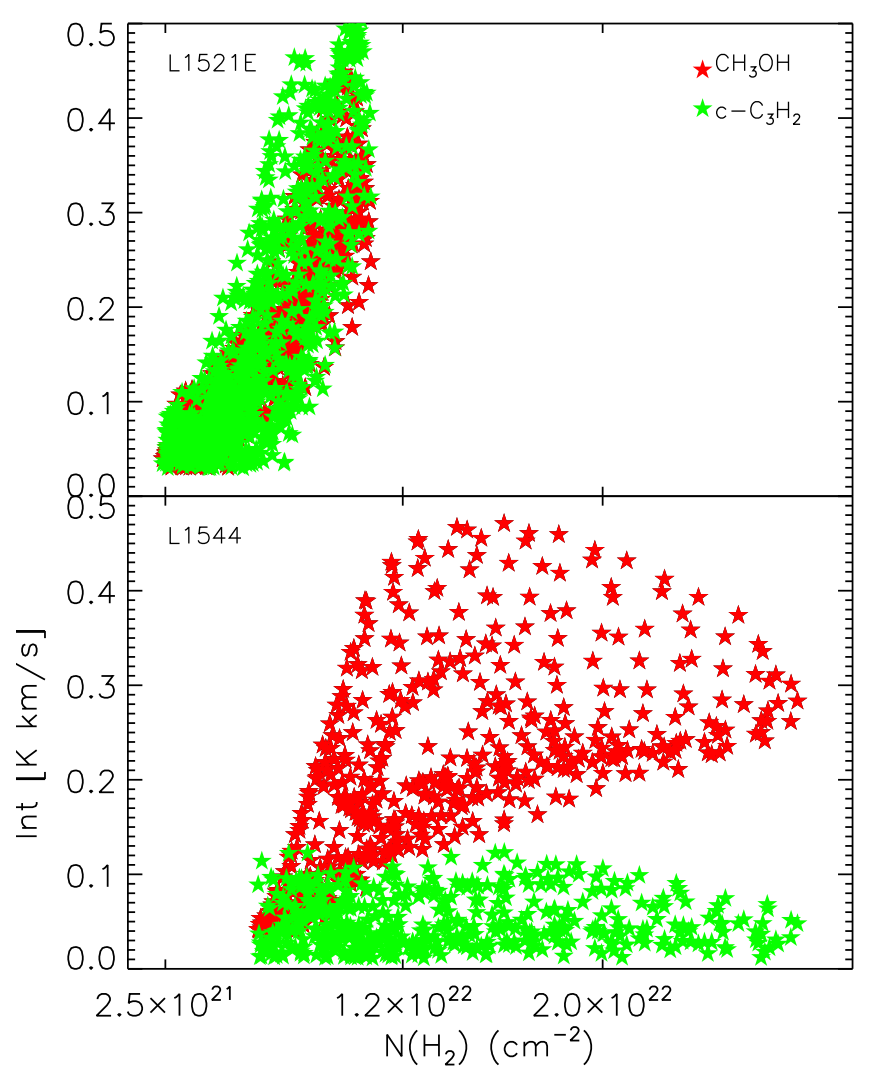

Fig. 12. Integrated intensities of $c-\mathrm{C}_{3} \mathrm{H}_{2}$ (green symbols) and $\mathrm{CH}_{3} \mathrm{OH}$ (red symbols) as function of $\mathrm{H}_{2}$ column densities toward L1521E and L1544. The data plotted for L1544 are from (Spezzano et al. 2016).

$\mathrm{CH}_{3} \mathrm{OH}$ intensities increase with increasing $N\left(\mathrm{H}_{2}\right)$, while $c-\mathrm{C}_{3} \mathrm{H}_{2}$ shows a flat intensity profile. This difference is due to the lower central density of L1521E compared to L1544 and the fact that the region of catastrophic CO freeze-out is smaller in L1521E compared to L1544.

- Comparing the relative abundances toward L1521E and $\mathrm{L} 1544$ we find that most sulfur bearing species such as $\mathrm{C}_{2} \mathrm{~S}$, $\mathrm{HCS}^{+}, \mathrm{C}^{34} \mathrm{~S}, \mathrm{C}^{33} \mathrm{~S}$, and $\mathrm{HCS}^{+}$are more abundant toward L1521E than toward L1544. This is confirmed by a chemical model that reproduces most column densities measured toward L1521E within an order of magnitude. This suggests that significant sulfur depletion is taking place during the dynamical evolution of dense cores.
Acknowledgements. The work by A.V. is supported by the Russian Science Foundation via the project 18-12-00351. M.T. acknowledges funding from project AYA2016-79 006-P.

\section{References}

Aikawa, Y., Ohashi, N., \& Herbst, E. 2003, ApJ, 593, 906

Andre, P., Ward-Thompson, D., \& Motte, F. 1996, A\&A, 314, 625

André, P., Men'shchikov, A., Bontemps, S., et al. 2010, A\&A, 518, L102

Caselli, P., Walmsley, C. M., Tafalla, M., Dore, L., \& Myers, P. C. 1999, ApJ, 523,165

Chacon-Tanarro, A., Pineda, J. E., Caselli, P., et al. 2019, A\&A, 623, A118

Crapsi, A., Caselli, P., Walmsley, C. M., et al. 2005, ApJ, 619, 379

Ford, A. B., \& Shirley, Y. L. 2011, ApJ, 728, 144

Frerking, M. A., Langer, W. D., \& Wilson, R. W. 1982, ApJ, 262, 590

Griffin, M. J., Abergel, A., Abreu, A., et al. 2010, A\&A, 518, L3

Hacar, A., Tafalla, M., Kauffmann, J., \& Kovács, A. 2013, A\&A, 554, A55

Henning, Th., Michel, B., \& Stognienko, R. 1995, Planet. Space Sci., 43, 1333

Hily-Blant, P., Walmsley, M., Pineau des Forêts, G., \& Flower, D. 2010, A\&A 513, A41

Hirota, T., Ito, T., \& Yamamoto, S. 2002, ApJ, 565, 359

Kirk, J. M., Ward-Thompson, D., \& André, P. 2007, MNRAS, 375, 843

Kong, S., Caselli, P., Tan, J. C., et al. 2015, ApJ, 804, 98

Laas, J. C., \& Caselli, P. 2019, A\&A, 624, A108

Lefloch, B., Bachiller, R., \& Ceccarelli, C. 2018, MNRAS, 477, 4792

Loinard, L., Mioduszewski, A. J., Rodríguez, L. F., et al. 2005, ApJ, 619, L179

Makiwa, G., Naylor, D. A., van der Wiel, M. H. D., et al. 2016, MNRAS, 458, 2150

Müller, H. S. P., Schlöder, F., Stutzki, J., \& Winnewisser, G. 2005, J. Mol. Struct., 742, 215

Penzias, A. A. 1981, ApJ, 249, 518

Pety, J. 2005, in EdP-Sciences Conference Series, eds. F. Casoli, T. Contini, J. Hameury, \& L. Pagani, SF2A-2005, 721

Pickett, H. M., Poynter, R. L., Cohen, E. A., et al. 1998, J. Quant. Spectr. Rad. Transf., 60, 883

Preibisch, Th., Ossenkopf, V., Yorke, H. W., \& Henning, Th. 1993, A\&A, 279, 577

Schöier, F. L., van der Tak, F. F. S., van Dishoeck, E. F., \& Black, J. H. 2005, A\&A, 432, 369

Spezzano, S., Bizzocchi, L., Caselli, P., Harju, J., \& Brünken, S. 2016, A\&A, 592, L11

Spezzano, S., Caselli, P., Bizzocchi, L., Giuliano, B. M., \& Lattanzi, V. 2017, A\&A, 606, A82

Swade, D. A. 1989, ApJ, 345, 828

Tafalla, M., \& Santiago, J. 2004, A\&A, 414, L53

Tafalla, M., Santiago-García, J., Myers, P. C., et al. 2006, A\&A, 455, 577

Torres, R. M., Loinard, L., Mioduszewski, A. J., \& Rodríguez, L. F. 2007, ApJ, 671,1813

Ungerechts, H., Bergin, E. A., Goldsmith, P. F., et al. 1997, ApJ, 482, 245

Vastel, C., Quénard, D., Le Gal, R., et al. 2018, MNRAS, 478, 5514

Vasyunin, A. I., Caselli, P., Dulieu, F., \& Jiménez-Serra, I. 2017, ApJ, 842, 33

Wakelam, V., \& Herbst, E. 2008, ApJ, 680, 371

Watanabe, N., \& Kouchi, A. 2002, ApJ, 571, 173

Wilson, T. L., \& Rood, R. 1994, ARA\&A, 32, 191

Yan, Q.-Z., Zhang, B., Xu, Y., et al. 2019, A\&A, 624, A6 


\section{Appendix A: The observed lines and their parameters}

Table A.1. Molecules and transitions detected toward L1521E.

\begin{tabular}{|c|c|c|c|c|c|c|c|c|c|c|c|}
\hline Molecule & Transition & $\begin{array}{r}v \\
(\mathrm{MHz})\end{array}$ & $\begin{array}{r}A_{i j} \\
\left(\mathrm{~s}^{-1}\right)\end{array}$ & $\begin{array}{l}E_{\text {up }} \\
(\mathrm{K})\end{array}$ & $\begin{array}{r}\int T_{\mathrm{MB}} \mathrm{d} V \\
\left(\mathrm{~K}^{-1} \mathrm{~km} \mathrm{~s}^{-1}\right)\end{array}$ & $\begin{array}{r}V_{\mathrm{LSR}} \\
\left(\mathrm{km} \mathrm{s}^{-1}\right)\end{array}$ & $\begin{array}{r}\Delta V \\
\left(\mathrm{~km} \mathrm{~s}^{-1}\right)\end{array}$ & $\begin{array}{r}T_{\text {peak }} \\
(\mathrm{K})\end{array}$ & $\begin{array}{l}3 \sigma \mathrm{rms} \\
(\mathrm{K})\end{array}$ & $\begin{array}{c}\text { Critical } \\
\text { density }\left(\mathrm{cm}^{-3}\right)\end{array}$ & $\begin{array}{l}\text { Detected toward } \\
\quad \text { L1544 }\end{array}$ \\
\hline $\mathrm{C}_{4} \mathrm{H}$ & $N=8-7, J=15 / 2-13 / 2, F=7-6 \& 8-7$ & 76156.0 & $1.82 \times 10^{-6}$ & 16.5 & $0.19 \pm 0.01$ & $6.76 \pm 0.01$ & $0.37 \pm 0.02$ & 0.47 & 0.13 & - & - \\
\hline $\mathrm{CH}_{3} \mathrm{CHO}^{*}$ & $4_{0,4}-3_{0,3}, E$ & 76866.4 & $1.43 \times 10^{-5}$ & 9.3 & $0.05 \pm 0.01$ & $6.79 \pm 0.04$ & $0.40 \pm 0.09$ & 0.12 & 0.03 & - & - \\
\hline $\mathrm{CH}_{3} \mathrm{CHO}$ & $4_{0,4}-3_{0,3}, A$ & 76879.0 & $1.43 \times 10^{-5}$ & 9.2 & $0.06 \pm 0.01$ & $7.07 \pm 0.03$ & $0.39 \pm 0.07$ & 0.15 & 0.03 & - & - \\
\hline $\mathrm{C}_{2} \mathrm{~S}$ & $N=6-5, J=6-5$ & 77731.7 & $2.03 \times 10^{-5}$ & 21.8 & $0.23 \pm 0.01$ & $6.77 \pm 0.01$ & $0.36 \pm 0.02$ & 0.62 & 0.18 & - & - \\
\hline $\mathrm{CC}^{34} \mathrm{~S}^{*}$ & $N=6-5, J=7-6$ & 79827.5 & $2.31 \times 10^{-5}$ & 15.1 & $0.05 \pm 0.01$ & $7.03 \pm 0.03$ & $0.32 \pm 0.05$ & 0.16 & 0.04 & - & - \\
\hline$l-\mathrm{C}_{4} \mathrm{H}_{2}^{*}$ & $9_{1,9}-8_{1,8}$ & 80046.7 & $4.69 \times 10^{-5}$ & 32.7 & $0.03 \pm 0.01$ & $6.81 \pm 0.05$ & $0.35 \pm 0.08$ & 0.08 & 0.01 & - & - \\
\hline $\mathrm{H}_{2} \mathrm{CCO}^{*}$ & $J_{K_{a} ; K_{c}}=4_{1,4}-3_{1,3}$ & 80076.7 & $5.04 \times 10^{-6}$ & 22.7 & $0.07 \pm 0.01$ & $7.04 \pm 0.02$ & $0.34 \pm 0.05$ & 0.20 & 0.05 & - & - \\
\hline $\mathrm{H}_{2} \mathrm{CCO}$ & $J_{K_{a} ; K_{c}}=4_{0,4}-3_{0,3}$ & 80832.1 & $5.52 \times 10^{-6}$ & 9.7 & $0.05 \pm 0.01$ & $6.93 \pm 0.07$ & $0.53 \pm 0.13$ & 0.09 & 0.03 & - & - \\
\hline $\mathrm{C}_{3} \mathrm{~S}^{*}$ & $J=14-13$ & 80928.2 & $3.86 \times 10^{-5}$ & 29.1 & $0.10 \pm 0.02$ & $6.89 \pm 0.02$ & $0.26 \pm 0.06$ & 0.35 & 0.07 & - & - \\
\hline $\mathrm{C}_{2} \mathrm{~S}^{*}$ & $N=6-5, J=7-6$ & 81505.2 & $2.46 \times 10^{-5}$ & 15.4 & $0.81 \pm 0.02$ & $6.71 \pm 0.01$ & $0.35 \pm 0.01$ & 2.18 & 0.35 & - & Yes \\
\hline $\mathrm{HC}_{3} \mathrm{~N}^{*}$ & $J=9-8$ & 81881.5 & $4.20 \times 10^{-5}$ & 19.7 & $0.80 \pm 0.01$ & $6.87 \pm 0.01$ & $0.33 \pm 0.01$ & 2.30 & 0.34 & $4.2 \times 10^{5}$ & Yes \\
\hline$c-\mathrm{C}_{3} \mathrm{H}_{2}^{*}$ & $J_{K_{a} ; K_{c}}=20,2-1_{1,1}$ & 82093.5 & $2.07 \times 10^{-5}$ & 6.4 & $0.41 \pm 0.01$ & $6.84 \pm 0.01$ & $0.33 \pm 0.01$ & 1.16 & 0.17 & $1.1 \times 10^{6}$ & Yes \\
\hline $\mathrm{OCS}^{*}$ & $J=7-6$ & 85139.1 & $1.71 \times 10^{-6}$ & 16.3 & $0.06 \pm 0.01$ & $6.82 \pm 0.04$ & $0.43 \pm 0.09$ & 0.13 & 0.04 & $2.3 \times 10^{4}$ & Yes \\
\hline $\mathrm{HC}^{18} \mathrm{O}^{+*}$ & $J=1-0$ & 85162.2 & $3.64 \times 10^{-5}$ & 4.1 & $0.07 \pm 0.01$ & $6.80 \pm 0.02$ & $0.33 \pm 0.05$ & 0.21 & 0.05 & $1.4 \times 10^{5}$ & Yes \\
\hline$c-\mathrm{C}_{3} \mathrm{H}_{2}$ & $J_{K_{a} ; K_{c}}=2_{1,2}-1_{0,1}$ & 85338.9 & $2.55 \times 10^{-5}$ & 6.5 & $0.81 \pm 0.01$ & $6.88 \pm 0.01$ & $0.39 \pm 0.01$ & 1.95 & 0.51 & $1.2 \times 10^{6}$ & Yes \\
\hline $\mathrm{HCS}^{+*}$ & $J=2-1$ & 85347.9 & $1.33 \times 10^{-5}$ & 6.1 & $0.24 \pm 0.01$ & $6.94 \pm 0.01$ & $0.27 \pm 0.02$ & 0.83 & 0.11 & $3.5 \times 10^{4}$ & Yes \\
\hline $\mathrm{CH}_{3} \mathrm{CCH}$ & $J_{K}=5_{1}-4_{1}$ & 85455.6 & $1.78 \times 10^{-6}$ & 19.5 & $0.16 \pm 0.01$ & $6.60 \pm 0.01$ & $0.34 \pm 0.02$ & 0.45 & 0.12 & - & Yes \\
\hline $\mathrm{CH}_{3} \mathrm{CCH}$ & $J_{K}=5_{0}-4_{0}$ & 85457.3 & $2.03 \times 10^{-6}$ & 12.3 & $0.18 \pm 0.01$ & $6.84 \pm 0.01$ & $0.34 \pm 0.02$ & 0.48 & 0.12 & - & Yes \\
\hline $\mathrm{C}_{4} \mathrm{H}^{*}$ & $N=9-8, J=19 / 2-17 / 2, F=10-9 \& 9-8$ & 85634.0 & $2.81 \times 10^{-6}$ & 20.6 & $0.15 \pm 0.01$ & $6.87 \pm 0.01$ & $0.31 \pm 0.03$ & 0.46 & 0.10 & - & Yes \\
\hline $\mathrm{C}_{4} \mathrm{H}$ & $N=9-8, J=17 / 2-15 / 2, F=9-8 \& 8-7$ & 85672.6 & $2.79 \times 10^{-6}$ & 20.6 & $0.14 \pm 0.01$ & $6.93 \pm 0.02$ & $0.27 \pm 0.02$ & 0.48 & 0.10 & - & Yes \\
\hline SO & $N_{J}=2{ }_{2}-1_{1}$ & 86094.0 & $5.25 \times 10^{-6}$ & 19.3 & $0.10 \pm 0.01$ & $7.06 \pm 0.02$ & $0.36 \pm 0.03$ & 0.24 & 0.05 & $8.2 \times 10^{4}$ & Yes \\
\hline $\mathrm{C}_{2} \mathrm{~S}$ & $N_{J}=7_{6}-6_{5}$ & 86181.4 & $2.78 \times 10^{-5}$ & 23.4 & $0.24 \pm 0.01$ & $6.83 \pm 0.01$ & $0.31 \pm 0.01$ & 0.71 & 0.14 & - & Yes \\
\hline $\mathrm{H}^{13} \mathrm{CN}$ & $J_{F}=1_{1}-0_{1}$ & 86338.8 & $2.22 \times 10^{-5}$ & 4.1 & $0.11 \pm 0.01$ & $7.06 \pm 0.03$ & $0.42 \pm 0.06$ & 0.24 & 0.10 & - & Yes \\
\hline $\mathrm{H}^{13} \mathrm{CN}^{*}$ & $J_{F}=1_{2}-0_{1}$ & 86340.2 & $2.22 \times 10^{-5}$ & 4.1 & $0.15 \pm 0.02$ & $6.95 \pm 0.03$ & $0.41 \pm 0.06$ & 0.34 & 0.10 & - & Yes \\
\hline $\mathrm{H}^{13} \mathrm{CN}$ & $J_{F}=1_{0}-0_{1}$ & 86342.3 & $2.22 \times 10^{-5}$ & 4.1 & $0.04 \pm 0.01$ & $6.97 \pm 0.03$ & $0.29 \pm 0.07$ & 0.14 & 0.10 & - & Yes \\
\hline $\mathrm{HCO}^{*}$ & $N_{K_{a}, K_{c}}=1_{0,1}-0_{0,0}, J=3 / 2-1 / 2, F=2-1$ & 86670.8 & $4.69 \times 10^{-6}$ & 4.2 & $0.07 \pm 0.01$ & $7.17 \pm 0.03$ & $0.39 \pm 0.06$ & 0.17 & 0.05 & - & Yes \\
\hline $\mathrm{HN}^{13} \mathrm{C}^{*}$ & $J=1-0$ & 87090.9 & $1.87 \times 10^{-5}$ & 4.2 & $0.31 \pm 0.04$ & $7.13 \pm 0.03$ & $0.58 \pm 0.08$ & 0.49 & 0.17 & - & Yes \\
\hline $\mathrm{C}_{2} \mathrm{H}$ & $N=1-0, J=3 / 2-1 / 2, F=1-1$ & 87284.2 & $3.75 \times 10^{-7}$ & 4.2 & $0.33 \pm 0.05$ & $6.98 \pm 0.02$ & $0.31 \pm 0.05$ & 0.98 & 0.20 & $7.5 \times 10^{4}$ & Yes \\
\hline $\mathrm{C}_{2} \mathrm{H}$ & $N=1-0, J=3 / 2-1 / 2, F=2-1$ & 87316.9 & $2.21 \times 10^{-6}$ & 4.2 & $0.79 \pm 0.05$ & $6.96 \pm 0.01$ & $0.40 \pm 0.03$ & 1.88 & 0.46 & $1.8 \times 10^{5}$ & Yes \\
\hline $\mathrm{C}_{2} \mathrm{H}$ & $N=1-0, J=3 / 2-1 / 2, F=1-0$ & 87328.6 & $1.83 \times 10^{-6}$ & 4.2 & $0.58 \pm 0.04$ & $7.00 \pm 0.01$ & $0.38 \pm 0.03$ & 1.46 & 0.34 & $2.3 \times 10^{5}$ & Yes \\
\hline $\mathrm{C}_{2} \mathrm{H}$ & $N=1-0, J=1 / 2-1 / 2, F=1-1$ & 87402.0 & $1.83 \times 10^{-6}$ & 4.2 & $0.44 \pm 0.05$ & $6.94 \pm 0.02$ & $0.33 \pm 0.04$ & 1.24 & 0.38 & $1.7 \times 10^{5}$ & Yes \\
\hline $\mathrm{C}_{2} \mathrm{H}^{*}$ & $N=1-0, J=1 / 2-1 / 2, F=0-1$ & 87407.2 & $2.22 \times 10^{-6}$ & 4.2 & $0.38 \pm 0.04$ & $6.87 \pm 0.01$ & $0.30 \pm 0.04$ & 1.18 & 0.21 & $1.4 \times 10^{5}$ & Yes \\
\hline $\mathrm{C}_{2} \mathrm{H}$ & $N=1-0, J=1 / 2-1 / 2, F=1-0$ & 87446.5 & $3.77 \times 10^{-7}$ & 4.2 & $0.21 \pm 0.04$ & $7.01 \pm 0.02$ & $0.26 \pm 0.09$ & 0.76 & 0.18 & $7.1 \times 10^{4}$ & Yes \\
\hline $\mathrm{HNCO}^{*}$ & $J_{K a, K c}=4_{0,4}-3_{0,3}$ & 87925.3 & $8.46 \times 10^{-6}$ & 10.6 & $0.21 \pm 0.04$ & $6.81 \pm 0.05$ & $0.54 \pm 0.12$ & 0.37 & 0.11 & $1.2 \times 10^{6}$ & Yes \\
\hline $\mathrm{HCN}$ & $J_{F}=1_{1}-0_{1}$ & 88630.4 & $2.43 \times 10^{-5}$ & 4.3 & $0.62 \pm 0.01$ & $6.86 \pm 0.01$ & $0.55 \pm 0.01$ & 1.06 & 0.41 & $4.3 \times 10^{6}$ & Yes \\
\hline $\mathrm{HCN}^{*}$ & $J_{F}=1_{2}-0_{1}$ & 88631.9 & $2.43 \times 10^{-5}$ & 4.3 & $0.85 \pm 0.08$ & $7.14 \pm 0.03$ & $0.49 \pm 0.06$ & 1.61 & 0.41 & $4.3 \times 10^{6}$ & Yes \\
\hline $\mathrm{HCN}$ & $J_{F}=1_{0}-0_{1}$ & 88633.9 & $2.43 \times 10^{-5}$ & 4.3 & $0.65 \pm 0.01$ & $6.73 \pm 0.01$ & $0.52 \pm 0.01$ & 1.19 & 0.41 & $4.3 \times 10^{6}$ & Yes \\
\hline $\mathrm{HCO}^{+*}$ & $J=1-0$ & 89188.5 & $4.19 \times 10^{-5}$ & 4.3 & $1.46 \pm 0.01$ & $6.86 \pm 0.01$ & $0.55 \pm 0.01$ & 2.50 & 0.79 & $1.6 \times 10^{5}$ & Yes \\
\hline $\mathrm{CH}_{3} \mathrm{CN}^{*}$ & $J_{K}=5_{1}-4_{1}$ & 91985.3 & $6.08 \times 10^{-5}$ & 20.4 & $0.03 \pm 0.01$ & $6.76 \pm 0.06$ & $0.38 \pm 0.10$ & 0.08 & 0.03 & $5.3 \times 10^{5}$ & Yes \\
\hline $\mathrm{CH}_{3} \mathrm{CN}$ & $J_{K}=5_{0}-4_{0}$ & 91987.1 & $6.33 \times 10^{-5}$ & 13.2 & $0.04 \pm 0.01$ & $6.98 \pm 0.05$ & $0.48 \pm 0.08$ & 0.09 & 0.03 & $5.4 \times 10^{5}$ & Yes \\
\hline $\mathrm{C}_{3} \mathrm{~S}$ & $J=16-15$ & 92488.5 & $6.13 \times 10^{-5}$ & 37.7 & $0.06 \pm 0.01$ & $6.91 \pm 0.03$ & $0.40 \pm 0.07$ & 0.13 & 0.03 & - & Yes \\
\hline${ }^{13} \mathrm{CS}^{*}$ & $J_{F}=2_{0}-1_{0}$ & 92494.3 & $1.41 \times 10^{-5}$ & 6.7 & $0.30 \pm 0.01$ & $6.95 \pm 0.01$ & $0.38 \pm 0.01$ & 0.73 & 0.16 & - & Yes \\
\hline $\mathrm{N}_{2} \mathrm{H}^{+}$ & $J=1-0, F_{1}=1-1, F=0-1$ & 93171.6 & $1.45 \times 10^{-6}$ & 4.5 & $0.04 \pm 0.01$ & $6.80 \pm 0.06$ & $0.40^{(b)}$ & 0.09 & 0.23 & $9.1 \times 10^{3}$ & Yes \\
\hline $\mathrm{N}_{2} \mathrm{H}^{+}$ & $J=1-0, F_{1}=1-1, F=2-2$ & 93171.9 & $6.89 \times 10^{-6}$ & 4.5 & $0.10 \pm 0.01$ & $6.83 \pm 0.02$ & $0.40^{(b)}$ & 0.24 & 0.23 & $1.7 \times 10^{5}$ & Yes \\
\hline $\mathrm{N}_{2} \mathrm{H}^{+}$ & $J=1-0, F_{1}=1-1, F=1-0$ & 93172.1 & $3.99 \times 10^{-6}$ & 4.5 & $0.07 \pm 0.01$ & $7.09 \pm 0.03$ & $0.40^{(b)}$ & 0.16 & 0.23 & $1.0 \times 10^{5}$ & Yes \\
\hline $\mathrm{N}_{2} \mathrm{H}^{+}$ & $J=1-0, F_{1}=2-1, F=2-1$ & 93173.5 & $6.53 \times 10^{-6}$ & 4.5 & $0.10 \pm 0.01$ & $6.95 \pm 0.01$ & $0.40 \pm 0.03$ & 0.24 & 0.23 & $1.6 \times 10^{5}$ & Yes \\
\hline $\mathrm{N}_{2} \mathrm{H}^{+}$ & $J=1-0, F_{1}=2-1, F=3-2$ & 93173.8 & $9.43 \times 10^{-6}$ & 4.5 & $0.16 \pm 0.01$ & $6.98 \pm 0.01$ & $0.41 \pm 0.03$ & 0.36 & 0.23 & $5.9 \times 10^{4}$ & Yes \\
\hline $\mathrm{N}_{2} \mathrm{H}^{+}$ & $J=1-0, F_{1}=2-1, F=1-1$ & 93174.0 & $3.99 \times 10^{-6}$ & 4.5 & $0.06 \pm 0.01$ & $7.02 \pm 0.03$ & $0.38 \pm 0.06$ & 0.15 & 0.23 & $8.9 \times 10^{5}$ & Yes \\
\hline $\mathrm{N}_{2} \mathrm{H}^{+*}$ & $J=1-0, F_{1}=0-1, F=1-2$ & 93176.3 & $3.99 \times 10^{-6}$ & 4.5 & $0.07 \pm 0.01$ & $6.91 \pm 0.02$ & $0.35 \pm 0.04$ & 0.19 & 0.23 & $2.2 \times 10^{5}$ & Yes \\
\hline $\mathrm{C}_{4} \mathrm{H}$ & $J=21 / 2-19 / 2, F=10-9 \& 11-10$ & 95150.4 & $3.60 \times 10^{-6}$ & 25.1 & $0.10 \pm 0.01$ & $6.93 \pm 0.01$ & $0.30 \pm 0.02$ & 0.32 & 0.07 & - & Yes \\
\hline $\mathrm{C}_{4} \mathrm{H}$ & $N=10-9, J=19 / 2-17 / 2, F=9-8 \& 10-9$ & 95189.0 & $3.58 \times 10^{-6}$ & 25.1 & $0.10 \pm 0.01$ & $7.03 \pm 0.02$ & $0.37 \pm 0.05$ & 0.24 & 0.07 & - & Yes \\
\hline $\mathrm{CH}_{3} \mathrm{CHO}$ & $5_{0,5}-4_{0,4},(E)$ & 95947.4 & $2.84 \times 10^{-5}$ & 13.9 & $0.03 \pm 0.01$ & $6.65 \pm 0.05$ & $0.40 \pm 0.05$ & 0.08 & 0.02 & - & Yes \\
\hline$C^{34} S^{*}$ & $J=2-1$ & 96412.9 & $1.61 \times 10^{-5}$ & 6.3 & $0.64 \pm 0.01$ & $6.86 \pm 0.01$ & $0.40 \pm 0.01$ & 1.51 & 0.30 & - & Yes \\
\hline $\mathrm{CH}_{3} \mathrm{OH}^{*}$ & $2_{-1,0}-1_{-1,0}\left(E_{2}\right)$ & 96739.3 & $2.56 \times 10^{-6}$ & 12.5 & $0.32 \pm 0.01$ & $6.67 \pm 0.01$ & $0.40 \pm 0.01$ & 0.76 & 0.20 & $2.9 \times 10^{4}$ & Yes \\
\hline $\mathrm{CH}_{3} \mathrm{OH}^{*}$ & $2_{0,2}-1_{0,1}\left(A^{+}\right)$ & 96741.4 & $3.41 \times 10^{-6}$ & 7.0 & $0.43 \pm 0.01$ & $6.93 \pm 0.01$ & $0.39 \pm 0.01$ & 1.05 & 0.20 & $3.1 \times 10^{4}$ & Yes \\
\hline$C^{33} S^{*}$ & $J=2-1, F=5 / 2-3 / 2,7 / 2-5 / 2, \& 1 / 2-1 / 2$ & 97171.8 & $1.63 \times 10^{-5}$ & 6.3 & $0.12 \pm 0.01$ & $6.83 \pm 0.01$ & $0.29 \pm 0.03$ & 0.39 & 0.08 & - & Yes \\
\hline
\end{tabular}

Notes. The line parameters are measured toward the Herschel dust peak using Gaussian fitting. Some of the detections toward L1544 are based on the spectrum measured in the $\sim 80-106 \mathrm{GHz}$ frequency range toward the dust peak of L1544 as part of ASAI (Lefloch et al. 2018; Vastel et al. 2018). The transitions marked with an asterisk were used to calculate column densities (Sect. 4.3) and for the principal component analysis (Sect. 4.2). ${ }^{(a)}$ The transitions which are marked with "_" were not covered in the available dataset for L1544. ${ }^{(b)}$ Fixed parameter in the fit. 
Z. Nagy et al.: The chemical structure of the very young starless core L1521E

Table A.1. continued.

\begin{tabular}{|c|c|c|c|c|c|c|c|c|c|c|c|}
\hline Molecule & Transition & $\begin{array}{r}v \\
(\mathrm{MHz}) \\
\end{array}$ & $\begin{array}{r}A_{i j} \\
\left(\mathrm{~s}^{-1}\right) \\
\end{array}$ & $\begin{array}{l}E_{\text {up }} \\
(\mathrm{K}) \\
\end{array}$ & $\begin{array}{r}\int T_{\mathrm{MB}} \mathrm{d} V \\
\left(\mathrm{~K} \mathrm{~km} \mathrm{~s}^{-1}\right) \\
\end{array}$ & $\begin{array}{r}V_{\mathrm{LSR}} \\
\left(\mathrm{km} \mathrm{s}^{-1}\right) \\
\end{array}$ & $\begin{array}{r}\Delta V \\
\left(\mathrm{~km} \mathrm{~s}^{-1}\right) \\
\end{array}$ & $\begin{array}{r}T_{\text {peak }} \\
(\mathrm{K}) \\
\end{array}$ & $\begin{array}{c}3 \sigma \mathrm{rms} \\
(\mathrm{K})\end{array}$ & $\begin{array}{c}\text { Critical } \\
\text { density }\left(\mathrm{cm}^{-3}\right)\end{array}$ & $\begin{array}{c}\text { Detected toward } \\
\text { L1544 }{ }^{(a)} \\
\end{array}$ \\
\hline${ }^{34} \mathrm{SO}^{*}$ & $N_{J}=2_{3}-1_{2}$ & 97715.4 & $1.09 \times 10^{-5}$ & 9.1 & $0.09 \pm 0.02$ & $6.81 \pm 0.02$ & $0.26 \pm 0.07$ & 0.32 & 0.03 & - & Yes \\
\hline $\mathrm{CS}^{*}$ & $J=2-1$ & 97981.0 & $1.70 \times 10^{-5}$ & 7.1 & $1.53 \pm 0.01$ & $7.00 \pm 0.01$ & $0.57 \pm 0.01$ & 2.51 & 0.92 & $3.4 \times 10^{5}$ & Yes \\
\hline $\mathrm{SO}^{*}$ & $J=3-2, N=2-1$ & 99299.9 & $1.15 \times 10^{-5}$ & 9.2 & $0.82 \pm 0.02$ & $6.92 \pm 0.01$ & $0.38 \pm 0.01$ & 2.05 & 0.39 & $2.8 \times 10^{5}$ & Yes \\
\hline $\mathrm{C}_{2} \mathrm{~S}$ & $N=8-7, J=7-6$ & 99866.5 & $4.47 \times 10^{-5}$ & 28.1 & $0.14 \pm 0.01$ & $6.84 \pm 0.01$ & $0.31 \pm 0.02$ & 0.41 & 0.09 & - & Yes \\
\hline $\mathrm{HC}_{3} \mathrm{~N}$ & $J=11-10$ & 100076.4 & $7.74 \times 10^{-5}$ & 28.8 & $0.43 \pm 0.01$ & $6.87 \pm 0.01$ & $0.36 \pm 0.01$ & 1.12 & 0.31 & $8.8 \times 10^{5}$ & Yes \\
\hline $\mathrm{H}_{2} \mathrm{CS}$ & $J_{K a, K c}=3_{1,3}-2_{1,2}$ & 101477.8 & $1.26 \times 10^{-5}$ & 22.9 & $0.63 \pm 0.01$ & $6.79 \pm 0.01$ & $0.33 \pm 0.01$ & 1.81 & 0.41 & $1.6 \times 10^{5}$ & Yes \\
\hline $\mathrm{H}_{2} \mathrm{CCO}$ & $J_{K a, K c}=5_{1,4}-4_{1,3}$ & 101981.4 & $1.09 \times 10^{-5}$ & 27.7 & $0.09 \pm 0.01$ & $6.77 \pm 0.02$ & $0.37 \pm 0.07$ & 0.24 & 0.04 & - & Yes \\
\hline $\mathrm{CH}_{3} \mathrm{CCH}$ & $J_{K}=6_{1}-5_{1}$ & 102546.0 & $3.17 \times 10^{-6}$ & 24.4 & $0.13 \pm 0.02$ & $6.91 \pm 0.03$ & $0.32 \pm 0.06$ & 0.38 & 0.10 & - & Yes \\
\hline $\mathrm{CH}_{3} \mathrm{CCH}^{*}$ & $J_{K}=6_{0}-5_{0}$ & 102548.0 & $3.26 \times 10^{-6}$ & 17.2 & $0.12 \pm 0.02$ & $6.79 \pm 0.02$ & $0.29 \pm 0.04$ & 0.40 & 0.10 & - & Yes \\
\hline $\mathrm{H}_{2} \mathrm{CS}^{*}$ & $J_{K a, K c}=3_{0,3}-2_{0,2}$ & 103040.5 & $1.48 \times 10^{-5}$ & 9.8 & $0.58 \pm 0.02$ & $6.85 \pm 0.01$ & $0.33 \pm 0.02$ & 1.65 & 0.22 & $1.4 \times 10^{5}$ & Yes \\
\hline $\mathrm{H}_{2} \mathrm{CS}$ & $J_{K a, K c}=3_{1,2}-2_{1,1}$ & 104617.0 & $1.38 \times 10^{-5}$ & 23.2 & $0.59 \pm 0.01$ & $6.75 \pm 0.01$ & $0.34 \pm 0.01$ & 1.65 & 0.38 & $2.3 \times 10^{5}$ & Yes \\
\hline $\mathrm{C}_{4} \mathrm{H}$ & $N=11-10, J=23 / 2-21 / 2, F=11-10 \& 12-11$ & 104666.6 & $4.83 \times 10^{-6}$ & 30.1 & $0.07 \pm 0.01$ & $6.95 \pm 0.03$ & $0.42 \pm 0.08$ & 0.16 & 0.04 & - & Yes \\
\hline $\mathrm{C}^{17} \mathrm{O}$ & $J=1-0 F=3 / 2-5 / 2$ & 112358.8 & $6.70 \times 10^{-8}$ & 5.4 & $0.12 \pm 0.02$ & $6.89 \pm 0.02$ & $0.31 \pm 0.05$ & 0.36 & 0.15 & $2.0 \times 10^{3}$ & - \\
\hline $\mathrm{C}^{17} \mathrm{O}$ & $J=1-0 F=7 / 2-5 / 2$ & 112359.0 & $6.70 \times 10^{-8}$ & 5.4 & $0.31 \pm 0.02$ & $6.86 \pm 0.01$ & $0.41 \pm 0.03$ & 0.71 & 0.15 & $2.0 \times 10^{3}$ & - \\
\hline $\mathrm{C}^{17} \mathrm{O}^{*}$ & $J=1-0 F=5 / 2-5 / 2$ & 112360.0 & $6.70 \times 10^{-8}$ & 5.4 & $0.24 \pm 0.01$ & $6.78 \pm 0.01$ & $0.42 \pm 0.03$ & 0.54 & 0.15 & $2.0 \times 10^{3}$ & - \\
\hline $\mathrm{CN}^{*}$ & $N=1-0, J=1 / 2-1 / 2, F=1 / 2-3 / 2$ & 113144.2 & $1.05 \times 10^{-5}$ & 5.4 & $0.27 \pm 0.02$ & $6.90 \pm 0.02$ & $0.46 \pm 0.03$ & 0.55 & 0.14 & $3.4 \times 10^{5}$ & - \\
\hline $\mathrm{CN}$ & $N=1-0, J=1 / 2-1 / 2, F=3 / 2-1 / 2$ & 113170.5 & $5.14 \times 10^{-6}$ & 5.4 & $0.21 \pm 0.02$ & $6.85 \pm 0.02$ & $0.40 \pm 0.04$ & 0.48 & 0.14 & $6.3 \times 10^{4}$ & - \\
\hline $\mathrm{CN}$ & $N=1-0, J=1 / 2-1 / 2, F=3 / 2-3 / 2$ & 113191.3 & $6.68 \times 10^{-6}$ & 5.4 & $0.26 \pm 0.02$ & $6.89 \pm 0.01$ & $0.44 \pm 0.03$ & 0.55 & 0.14 & $1.3 \times 10^{5}$ & - \\
\hline
\end{tabular}

